Marcelo Fernando Matielo

\title{
Incidência de trombose venosa profunda pós- operatória no membro amputado de pacientes com doença arterial oclusiva periférica
}

Tese apresentada à Faculdade de Medicina da Universidade de São Paulo para obtenção de título de Doutor em Ciências

Área de concentração: Clínica Cirúrgica Orientador: Dr. Calógero Presti

São Paulo

2008 


\section{Dados Internacionais de Catalogação na Publicação (CIP)}

Preparada pela Biblioteca da

Faculdade de Medicina da Universidade de São Paulo Creprodução autorizada pelo autor

Matielo, Marcelo Fernando

Incidência de trombose venosa profunda pós-operatória no membro amputado de pacientes com doença arterial oclusiva periférica / Marcelo Fernando Matielo. - São Paulo, 2008.

Tese (doutorado)--Faculdade de Medicina da Universidade de São Paulo. Departamento de Cirurgia.

Área de concentração: Clínica Cirúrgica.

Orientador: Calógero Presti.

Descritores: 1.Trombose venosa/diagnóstico 2.Amputação 3.Complicações pósoperatórias 4.Ecocardiografia Doppler 5.Incidência 


\section{DEDICATÓRIA}

Aos meus pais, Celso e Estela, pelo amor incondicional, pelos ensinamentos de caráter e virtude, por acreditarem sempre em mim.

Ao meu filho, Diego, razão pura da minha existência, pelas horas que deixei de lhe dedicar para que eu pudesse realizar meu sonho. 


\section{AGRADECIMENTOS}

Ao professor Doutor Pedro Puech-Leão, professor titular da Disciplina de Cirurgia Vascular da FMUSP, pela oportunidade, confiança, colaboração e apoio para o desenvolvimento deste projeto.

Minha gratidão ao Prof. Dr. Calógero Presti, meu orientador, pela dedicação, ensinamentos e aconselhamento inestimável em cada detalhe deste projeto.

Ao Dr. Baptista Muraco Netto, professor assistente doutor da Disciplina de Cirurgia Vascular da FMUSP, pela sugestão do assunto do projeto e aconselhamento inestimável, tanto para o projeto como para a vida profissional.

Ao Professor Doutor Ricardo Aun, professor associado da Disciplina de Cirurgia Vascular da FMUSP e responsável pelo pronto-socorro de Cirurgia Vascular, pelo apoio e encorajamento neste projeto.

Ao Dr. Marcos Menezes, por sua dedicação e indispensável apoio na realização dos exames ecográficos dos pacientes..

Ao amigo Dr. Ivan Benaduce Casella, assistente do HC/FMUSP, pela análise estatística, aconselhamento e apoio em todas as etapas deste projeto.

Aos professores e médicos assistentes do Serviço de Cirurgia Vascular do HC/FMUSP, pelo convívio e receptividade apresentada.

Aos médicos residentes do Serviço de Cirurgia Vascular do HC/FMUSP, pelo indispensável auxílio na realização deste projeto.

Aos amigos e professores do Serviço de Cirurgia Vascular do Hospital do Servidor Público Estadual de São Paulo, Drs José Gonzalez, Luiz Carlos Bérgamo, Chirstiano S. Pecêgo, Francisco Cardoso Brochado Neto, Roberto Sacilotto e 
Katsumi Nakano por todo meu aprendizado na especialidade, pela amizade e estímulo para realização deste projeto.

Aos amigos do Serviço de Cirurgia Vascular do Hospital do Servidor Público Estadual de São Paulo, Drs Marcos Godoy, Edson Nakamura, Sergio Tiossi, Ronaldo Daudt e médicos residentes pela colaboração e apoio em nosso dia a dia de trabalho..

À fisioterapeuta Shirley Nakayama, pelo apoio, compreensão e dedicação durante a elaboração desta tese.

Aos funcionários da secretaria e do ambulatório da Cirurgia Vascular, e do setor de Radiologia do pronto-socorro, pelo apoio e carinho recebido por todos.

Aos pacientes, seus cônjuges e parentes, pela confiança em mim depositada.

Aos amigos conhecidos e desconhecidos que de alguma forma apoiaram este projeto. 


\section{SUMÁRIO}

\section{LISTAS}

LISTA DE ABREVIATURAS viii

LISTA DE SIGLAS viii

LISTA DE QUADROS Ix

LISTA DE TABELAS Ix

LISTA DE GRÁFICOS X X

$\begin{array}{ll}\text { RESUMO } & \mathrm{Xi}\end{array}$

SUMMARY Xii

\section{INTRODUÇÃO}

1.1 Trombose venosa profunda - Considerações Gerais 2

1.2 Métodos Diagnósticos da TVP 4

1.3 Tratamento da TVP 6

1.4 Doença Arterial Obstrutiva Periférica e Amputação Maior $\quad 7$

1.5 TVP e amputação maior de membro inferior 9 


\section{MÉTODO}

3.1 Critérios de Inclusão 16

3.2 Critérios de não Inclusão 16

$\begin{array}{ll}3.3 \text { Critérios de exclusão } & 17\end{array}$

3.4 Variáveis Pré-operatórias 17

$\begin{array}{ll}3.5 \text { Variáveis cirúrgicas } & 19\end{array}$

3.6 Variáveis pós-operatórias 20

$\begin{array}{ll}3.7 \text { Eco-Doppler } & 21\end{array}$

$\begin{array}{ll}3.8 \text { Tratamento } & 22\end{array}$

3.9 Cálculo da Amostra 23

3.10 Análise estatística $\quad 25$

4. RESULTADOS 27

5. DISCUSSÃO 35

6. CONCLUSÕES 45

7. ANEXOS

$\begin{array}{ll}\text { ANEXO A } & 47\end{array}$

$\begin{array}{ll}\text { ANEXO B } & 48\end{array}$

8. REFERÊENCIAS 


\section{APÊNDICE}

\section{LISTA DE ABREVIATURAS}

$\begin{array}{ll}\text { ui } & \text { unidades internacionais } \\ \text { h } & \text { hora } \\ \text { n } & \text { número } \\ < & \text { menor } \\ > & \text { igual ou maior } \\ \text { et al } & \text { e outros } \\ \text { ep } & \text { erro padrão }\end{array}$

\section{LISTA DE SIGLAS}

$\begin{array}{ll}\text { TEV } & \text { tromboembolismo venoso } \\ \text { TVP } & \text { trombose venosa profunda } \\ \text { EP } & \text { embolia pulmonar } \\ \text { SUS } & \text { sistema único de saúde } \\ \text { HNF } & \text { heparina não fracionada } \\ \text { HBPM } & \text { heparina de baixo peso molecular } \\ \text { DAOP } & \text { doença arterial obstrutiva periférica } \\ \text { FMUSP } & \text { Faculdade de Medicina da Universidade de São Paulo } \\ \text { HCFMUSP } & \text { Hospital das Clínicas Faculdade de Medicina da Universidade } \\ & \text { de São Paulo }\end{array}$




$\begin{array}{ll}\text { EUA } & \text { Estados Unidos da América } \\ \text { Masc } & \text { masculino } \\ \text { Fem } & \text { feminino } \\ \text { Indiv } & \text { indivíduos } \\ \text { AVC } & \text { acidente vascular cerebral }\end{array}$

\section{LISTA DE QUADROS}

$\begin{array}{lll}\text { Quadro 1 Doenças associadas } & 18\end{array}$

$\begin{array}{llr}\text { Quadro } 2 & \text { Fatores de risco para TVP avaliados } & 18\end{array}$

Quadro 3 Nível de oclusão conforme a palpação dos pulsos 19

Quadro 4 Complicações no coto de amputação 20

Quadro 5 Outras complicações pós-operatórias 21

\section{LISTA DE TABELAS}

$\begin{array}{lll}\text { Tabela } 1 \text { Causas de exclusão dos pacientes do estudo } & 27\end{array}$

Tabela 2 Prevalência de Doenças Associadas 27

Tabela 3 Causas de necrose de coto e nível de amputação 28

Tabela 4 Segmento venoso acometido e nível de amputação 29

Tabela 5 Análise univariável de fatores de risco para trombose venosa profunda de coto de amputação 


\section{LISTA DE GRÁFICOS}

Gráfico 1 Incidência cumulativa de trombose venosa de coto de amputação

Gráfico 2 Incidência cumulativa de trombose venosa de coto de amputação dividida pelo nível de amputação

Gráfico 3 Incidência cumulativa de trombose venosa de coto de amputação dividida pela faixa etária 


\section{RESUMO}

Matielo MF. Incidência de trombose venosa profunda pós-operatória no membro amputado de pacientes com doença arterial oclusiva periférica [tese]. São Paulo: Faculdade de Medicina, Universidade de São Paulo; 2008. 61p.

Introdução: Pacientes submetidos à amputação de membro inferior por doença arterial obstrutiva periférica (DAOP) estão em risco para o desenvolvimento de trombose venosa profunda (TVP). Há poucos estudos na literatura sobre a incidência no pós-operatório precoce e quanto aos fatores de risco no desenvolvimento da TVP no membro amputado. Objetivo: A finalidade deste estudo é avaliar, de modo prospectivo, a incidência de trombose venosa profunda pós-operatória em até 35 dias, em pacientes submetidos à amputação de membro inferior por doença arterial obstrutiva periférica, sua relação com comorbidades e com óbito. Método: De setembro de 2004 a março de 2006, foram estudados 56 pacientes ( 29 homens; média de idade 67,25 anos) submetidos a 62 amputações (36 transtibiais e 26 transfemorais), utilizando-se eco-Doppler no pré-operatório e aproximadamente no $7^{\circ}$ e $31^{\circ}$ dia de pós-operatório. Resultado: Houve TVP em 16 (25,8\%) membros amputados, sendo 10 casos em amputações transfemorais e 6 casos em transtibiais. A incidência cumulativa no período até 35 dias foi de $28 \%$ ( Kaplan-Meier ). Houve diferença significativa na incidência de TVP entre amputações transfemorais (37,5\%) e transtibiais $(21,2 \%), \mathrm{p}=0,04$. Outro fator de risco para TVP foi idade igual ou superior a 70 anos $(48,9$ vs $16,8 \%, p=0,021)$. Houve 01 caso de embolia pulmonar sintomática não fatal em paciente com TVP já diagnosticada. Não houve relação entre outras comorbidades e TVP. A trombose venosa no membro amputado não influenciou na taxa de óbito que foi de 9,7\%. Conclusões: A incidência de TVP no pós-operatório recente (até 35 dias) foi elevada principalmente em pacientes com idade igual e superior a 70 anos e nas amputações transfemorais. Os pacientes com DAOP submetidos a grandes amputações devem ser considerados de alto risco para TVP, mesmo após alta hospitalar. 


\section{SUMMARY}

Matielo MF. Incidence of postoperative deep venous thrombosis in amputated lower extremity of patients with peripheral arterial disease [thesis]. São Paulo: "Faculdade de Medicina, Universidade de São Paulo"; 2008. 61p.

Introduction: Patients undergoing amputation of the lower limb due to Peripheral Arterial Disease (PAD) are at risk for developing Deep Venous Thrombosis (DVT). There are few studies in the research literature on the incidence of DVT during the early postoperative period and the risk factors for the development of DVT in the amputation stump. Objective: The goal of this prospective study was to evaluate the incidence of deep venous thrombosis during the first 35 postoperative days in patients who had undergone amputation of the lower extremity due to PAD, and its relation to comorbidities and death. Method: From September 2004 to March 2006, fifty-six patients (29 men, mean age 67.25 years) underwent 62 amputations (36 below knee amputation - BKA and 26 above knee amputation- AKA), and echoDoppler scanning on preoperative, and approximately the seventh and $31^{\text {st }}$ postoperative days. Results: DVT occurred in $16(25.8 \%)$ of the amputated extremities, (10 AKA and $06 \mathrm{BKA}$ ). The cumulative incidence in the 35 day postoperative period was $28 \%$ (Kaplan-Meier). There was a significant difference in the incidence of DVT between AKA (37.5\%) and BKA (21.2\%), p =.04. Another DVT risk factor was age equal to or above 70 years $(48.9$ vs. $16.8 \%, p=.021)$. There was one case of symptomatic non-fatal pulmonary embolism in a patient already diagnosed with DVT. There was no relation between other comorbidities and DVT. Venous Thrombosis in the amputation stump did not influence the mortality rate which was $9.7 \%$. Conclusions: The incidence of DVT in the early post-operative period (up to 35 days) was elevated mainly in patients 70 years of age or older and in AKA. Patients with PAD who have recently undergone major amputations should be considered at high risk for DVT, even after hospital discharge. 
1. INTRODUÇÃO 
O tromboembolismo venoso (TEV) é uma doença que inclui a trombose venosa profunda (TVP) e embolia pulmonar (EP). Pode produzir sintomas pouco específicos, muitas vezes dificultando o diagnóstico. É de extrema importância pela sua elevada freqüência, morbidade e mortalidade.

\subsection{Trombose Venosa Profunda - Considerações Gerais}

Cerca de 2.000.000 de pessoas ao ano desenvolvem TEV e, aproximadamente, 100.000 morrem por EP nos Estados Unidos da América. Estimase que entre 250.000 a 500.000 pessoas, ao ano, apresentem TEV sintomático nos Estados Unidos da América, sendo que, em menos de 50\% dos casos há sintomas de TVP e, em conseqüência, o diagnóstico, profilaxia e tratamento adequados não são realizados. $^{1,2}$

No Brasil, Castro-Silva fez uma projeção de 68.000 internações ao ano pelo SUS em conseqüência de TEV, sendo que $15,2 \%$ desses pacientes evoluem a óbito. ${ }^{3}$

Stein et al., ${ }^{4}$ em estudo sobre a incidência de TVP em pacientes internados em hospital geral, evidenciaram em 44.136 internações consecutivas 271 casos de TVP em pacientes que receberam ou não algum tipo de profilaxia e, desses, 57 apresentaram EP. Estima-se que, em apenas um quarto a um quinto dos pacientes com internações recentes (até 30 dias), tenha sido diagnosticado corretamente o TEV. ${ }^{5-7}$

São considerados principais fatores de risco para TEV em pacientes cirúrgicos: idade superior a 40 anos, TVP e EP prévias, tempo operatório maior que 60 minutos, obesidade, insuficiência venosa, cardiopatia, presença de processo 
infeccioso, uso de estrógeno, imobilização prolongada, acidente vascular cerebral, paralisia, presença de tumores malignos, trombofilia, cirurgia abdominal, ortopédica, ginecológica, urológica e vascular. ${ }^{2,6,8-10}$

Fowkes et al., ${ }^{11}$ em artigo de revisão da literatura, evidenciaram um risco de TVP 8 vezes maior na população hospitalar e 22 vezes maior nos pacientes cirúrgicos quando comparados com a população em geral.

Em outro artigo de revisão da literatura onde foi estudada a história natural de TEV, Kearon ${ }^{12}$ relata que a maior parte dos pacientes com TVP, ela se inicia em veias infrapoplíteas em especial em veias musculares e ocorre no momento da cirurgia, sendo que metade dos pacientes se resolve espontaneamente em 72 horas e que em apenas 1/6 dos casos ocorre extensão proximal para as veias tronculares. Não observou nenhum caso de EP nos pacientes que apresentavam TVP em veias infrapoplíteas, sem extensão proximal, enquanto que nos casos de TVP proximal houve uma incidência de 40 a 50\% de EP assintomáticas, diagnosticadas com cintilografia de ventilação e perfusão. Refere ainda que em $61 \%$ dos pacientes cirúrgicos a TVP ocorre após o $3^{\circ}$ dia de pós-operatório, em $9 \%$ após uma semana, e em alguns casos podendo ocorrer até meses após o procedimento cirúrgico.

A TVP e EP, no pós-operatório do paciente cirúrgico, são de extrema importância prática. A necessidade do diagnóstico preciso e precoce da TVP é fundamental para o bom resultado terapêutico e prevenção da EP e suas graves conseqüências. 


\subsection{Métodos Diagnósticos da TVP}

O diagnóstico clínico da TVP é bastante falho: há grandes chances de não se fazer diagnóstico em pacientes assintomáticos e fazer diagnóstico equivocado em pacientes sintomáticos, pois existem outras doenças que podem ter os mesmos

sintomas que a TVP. ${ }^{13,14}$ Nos últimos anos, avanços tecnológicos tornaram disponíveis exames de imagem para o rastreamento da TVP, com diferentes benefícios, riscos e custos.

O método das plaquetas autógenas marcadas com Indium-111 é capaz de diagnosticar TVP em 77\% dos pacientes sintomáticos e, segundo alguns autores, o método demonstra o envolvimento das plaquetas na fisiopatologia da TVP e pode ser útil na monitorização dos efeitos da anticoagulação. ${ }^{15}$

Lennox et al., ${ }^{16}$ utilizando a dosagem de Dímero D para diagnóstico de TVP em pacientes com TVP sintomáticos, obtiveram $91,3 \%$ de sensibilidade, $81,9 \%$ de especificidade e valor preditivo negativo de 96,6\%, porém apresentando desvantagem de grande número de falsos positivos quando há concomitância de infecção, cirurgia recente e outras doenças clínicas.

Em artigo de revisão da literatura, Weinmann e Salzman ${ }^{8}$ comparam a eficácia de vários métodos em relação à flebografia. Verificaram que a pletismografia por impedância tem sensibilidade de $92 \%$ e especificidade de $95 \%$ para pacientes com sintomas de TVP, e sensibilidade de $22 \%$ e especificidade de 98\% para os sem sintomas, não sendo adequada para detectar trombos em veias de perna e trombos não oclusivos. 
A pletismografia a ar tem baixo custo, é exame não invasivo, de fácil realização, mas de pouco valor no diagnóstico de trombos não oclusivos. ${ }^{17}$

A flebografia é o padrão ouro com sensibilidade próxima a $100 \%$. No entanto tem como inconveniente ser exame invasivo, usar contraste iodado com eventuais complicações relacionadas à nefrotoxidade, alergia e a fenômenos trombóticos venosos por agressão ao endotélio. ${ }^{18}$

A angioressonância nuclear magnética também pode ser utilizada para o diagnóstico de TVP com sensibilidade de 96\% e especificidade de 92\%, mas apresenta limitações importantes como a dificuldade de uso em pacientes com claustrofobia ou com próteses metálicas, necessidade de tempo adequado para aquisição do contraste e exame demorado. ${ }^{19}$

A venografia, por tomografia helicoidal, apresenta sensibilidade e especificidade de $94,5 \%$ e $98,2 \%$ respectivamente. É exame que pode ser realizado conjuntamente com a aquisição da venografia de membros inferiores e o estudo das artérias pulmonares para o diagnóstico de EP. Tem como desvantagens o uso de contraste iodado, radiação ionizante e não ser um equipamento disponível em todos os hospitais. ${ }^{20}$

Meissner ${ }^{21}$ refere o eco-Doppler colorido como bom método para a investigação da TVP e Zierler ${ }^{22}$ observou sensibilidade e especificidade de $95 \%$ para casos de TVP em pacientes sintomáticos.

Mattos et al., ${ }^{23}$ usando eco-Doppler para estudo de TVP em pacientes assintomáticos, obtiveram valor preditivo negativo de $88 \%$, e um valor preditivo positivo de $89 \%$ quando analisados todos os segmentos venosos. Quando excluídas as veias de perna, os valores preditivos positivos passaram a 97\% e 100\%, 
respectivamente para os pacientes sintomáticos e assintomáticos, com sensibilidade de $100 \%$ e especificidade de $98 \%$ para pacientes sintomáticos, sensibilidade de $67 \%$ e especificidade de $100 \%$ para assintomáticos. Enquanto Elias et al. ${ }^{24}$ evidenciaram uma sensibilidade e especificidade de $94 \%$ para pacientes assintomáticos diagnosticados para o segmento proximal, e sensibilidade e especificidade de $89 \%$ para assintomáticos quando o segmento comprometido era somente o distal.

Utilizando o eco-Doppler, Lohr et al. ${ }^{25}$ evidenciaram incidência elevada de TVP bilateral em pacientes com suspeita clínica em apenas um dos membros inferiores.

Zierler ${ }^{26}$ afirma que o eco-Doppler é atualmente o exame de escolha para a rotina na investigação diagnóstica de TVP em membros inferiores, seja no segmento proximal ou distal. É um exame não invasivo, não usa radiação ionizante, não utiliza contraste iônico, é reprodutível e de baixo custo.

\subsection{Tratamento da TVP}

A importância do tratamento adequado da TVP consiste na parada da propagação e recorrência do trombo e, principalmente, em evitar a ocorrência de EP. 27 A essência de qualquer estratégia é para identificar principalmente a TVP proximal, na expectativa de que o início da anticoagulação no estágio pré sintomático possa prevenir EP fatal. ${ }^{28}$

O tempo de tratamento da TVP proximal depende de alguns fatores como: pós-operatório recente, problemas clínicos recentes transitórios e outros. Nesses casos em que há um fator desencadeante bem estabelecido para o desenvolvimento 
da TVP, e é transitório, o tempo de anticoagulação pode ser por um período de 3 meses segundo alguns autores. ${ }^{29-31}$ Quando há presença de outros fatores como cardiopatias, cateter venoso central prévio, seqüela de acidente vascular cerebral e outros, a anticoagulação deve ser mantida por, pelo menos, 6 meses. ${ }^{32-34}$

A necessidade de anticoagulação, nos casos de TVP distal, é controversa; Lagerdstedt et al., ${ }^{35}$ estudando os casos sintomáticos de TVP distal, evidenciaram propagação do trombo proximal nos pacientes sem tratamento de $17,8 \%$ e, entre esses, houve apenas um caso de EP não fatal. Labropoulos et al. ${ }^{36}$ evidenciaram uma progressão proximal do trombo em $13 \%$ dos pacientes, e também somente em um caso houve EP não fatal. Observaram ainda que não houve diferença na propagação do trombo entre os pacientes anticoagulados e naqueles em que não se optou pelo seu uso. Pinede et al. ${ }^{37}$ evidenciaram que o tratamento por 6 ou 12 semanas na TVP distal são equivalentes quanto à taxa de EP, recorrência da TVP e riscos de sangramento.

Independente do segmento venoso acometido se indica a anticoagulação, deve-se iniciar o tratamento com uso de heparina não fracionada (HNF) ou de baixo peso molecular (HBPM) e, conjuntamente, o anticoagulante oral, quando não há contra-indicações a esse tratamento. ${ }^{38}$

\subsection{Doença Arterial Obstrutiva Periférica e Amputação Maior}

A aterosclerose é a principal causa de doença arterial obstrutiva periférica (DAOP) ${ }^{39}$ e a prevalência da doença assintomática é estimada entre $0.9 \%$ a $22 \%$ da população. A proporção de pacientes sintomáticos com claudicação intermitente, 
para os assintomáticos, é de 1:0.9 a 1:6.0. Dentre os pacientes com claudicação intermitente, estima-se que $3 \%$ a $5 \%$ irão desenvolver isquemia crítica (dor em repouso e/ou lesão trófica) em 5 anos e, desses, $25 \%$ serão submetidos à amputação maior de membro inferior. ${ }^{40}$

Há estimativa populacional de 500 a 1.000 casos de amputações maiores por 1.000.000 de pessoas ao ano nos Estados Unidos da América. ${ }^{39,41}$ Em estudo brasileiro no município do Rio de Janeiro, Spichler et al. ${ }^{42}$ obtiveram uma incidência de 400 amputações em decorrência de DAOP por 1.000.000 de pessoas ao ano.

As indicações das amputações maiores em geral são devidas a gangrenas úmidas, gangrenas secas, úlceras isquêmicas e dor em repouso, ${ }^{43} \mathrm{em}$ pacientes com insucesso das revascularizações arteriais ou quando as lesões isquêmicas são tão extensas que não há indicação de revascularização. ${ }^{44,45}$ As doenças associadas e fatores de risco mais freqüentes nos pacientes submetidos às amputações maiores são o Diabetes Mellitus, hipertensão arterial sistêmica, cardiopatias, tabagismo e doença cerebrovascular. ${ }^{46}$

O critério para indicação do nível de amputação geralmente é clínico, levando-se em consideração a localização da oclusão arterial, temperatura da pele no local em que se pretende realizar a amputação, extensão da infecção ou gangrena, condições clínicas do doente e se há perspectiva de reabilitação. ${ }^{47,48}$

A amputação transtibial é o tipo de amputação mais realizada mesmo com ausência de pulso poplíteo palpável. ${ }^{49,50}$ A amputação transtibial deve ser realizada mesmo após oclusão da revascularização distal, pois esta não influencia na perda de nível. ${ }^{51,52}$ 
A mortalidade operatória das grandes amputações varia de 0 a $16 \%,{ }^{53,54}$ sendo maior nas amputações transfemorais por serem indicadas em doentes mais idosos e com maiores associações de comorbidades. ${ }^{51}$

\subsection{TVP e amputação maior de membro inferior}

Apesar dos inúmeros estudos existentes quanto à incidência de TVP e EP no paciente cirúrgico, poucas são as referências e estudos nos pacientes submetidos à amputação cirúrgica das extremidades inferiores.

O primeiro estudo relatado na literatura foi o de Harper et al. ${ }^{55}$ em 1973 . O autor relatou uma incidência de TVP iliacofemoral em 66,7\% dos pacientes submetidos à amputação de coxa sem nenhum tipo de profilaxia e em $8,3 \%$ dos pacientes em que se usou de Dextran 70 . Neste estudo, um cateter era mantido na veia safena magna do coto de amputação durante o pós-operatório para realização de flebografias, para o diagnóstico da TVP.

Williams et al. ${ }^{56}$ evidenciaram, no intra-operatório de amputação do membro inferior, a presença de TVP em 11\% dos casos, com incidência de EP em 100\% desses pacientes, mas não realizaram nenhum estudo no pós-operatório para verificar os casos assintomáticos de TVP.

Em contrapartida, Barnes ${ }^{57}$ não encontrou nenhum caso de TVP nos pacientes submetidos à amputação de membros inferiores quando estudados com Doppler de onda contínua.

Novamente Willians et al., ${ }^{58}$ em 1978, estudando a incidência somente de EP (cintilografia e/ou autópsia) em amputados, evidenciaram um risco quatro vezes 
maior de EP em amputações transfemorais em relação às transtibiais, não havendo diferença da ocorrência do evento nos que foram medicados com HNF (5.000 ui 12/12 h) ou solução salina.

Em um estudo de Fletcher e Batiste, ${ }^{59}$ foi observada incidência de TVP de 9,1\% em pacientes submetidos à revascularização aorto-ilíaca ou infrainguinal. Para as amputações a incidência foi de 14,3\%, apesar do uso preventivo de heparina e compressão pneumática intermitente dos membros inferiores. Os autores não especificam se o diagnóstico das tromboses foi no pré ou pós-operatório e se puderam realizar estudo em todos os pacientes no pós-operatório, visto que a mortalidade operatória foi $23,8 \%$ no grupo de amputados.

Em estudo prospectivo, Yeager et al., ${ }^{10}$ empregando o eco-Doppler no pré-operatório e no pós-operatório de 72 pacientes submetidos a 82 amputações maiores, observaram a presença de TVP em 9 pacientes, sendo 4 casos de TVP no membro contralateral, 01 bilateral e 4 no membro amputado. Desses, 6 apresentaram TVP no pré-operatório e 3 no pós-operatório. Dos 4 pacientes que apresentaram TVP no membro amputado, não refere quando foram realizados os diagnósticos, se no pré ou pós-operatório. Dos 3 pacientes com diagnóstico de TVP no pós-operatório, não refere se é no coto de amputação, e um não havia realizado estudo no pré-operatório. Nesse estudo, ele não especifica em que época do pós-operatório realizou o estudo com o eco-Doppler, referindo apenas ser antes da alta hospitalar. Além disso, 9 pacientes estavam na vigência de anticoagulação plena antes e depois do procedimento cirúrgico e não foram excluídos do estudo. Ainda nesse estudo, 12 pacientes não realizaram eco-Doppler no pré-operatório e 2 pacientes não realizaram estudo no pós-operatório. 
Em estudo com amputações maiores dos membros inferiores, Muraco et al. ${ }^{60}$ realizaram eco-Doppler no pré-operatório e no terceiro ao sétimo dia de pósoperatório e evidenciaram a presença de TVP no coto de amputação em 8 dos 22 casos, sendo 3 em amputações transtibiais e 5 transfemorais.

Outro estudo prospectivo realizado no Brasil por Lastória et al., ${ }^{61}$ em 75 pacientes submetidos a amputações maiores de membros inferiores por DAOP e randomizados para profilaxia com HNF (34) e HBPM (41), através de realização de eco-Doppler no pré-operatório e no pós-operatório entre o quinto ao oitavo dia, evidenciaram a incidência de $8(10,7 \%)$ casos de TVP, não havendo diferença significativa na incidência entre os dois grupos. Também não evidenciaram diferença na incidência de TVP quanto ao sexo e nível da amputação.

Zickler et al., ${ }^{62}$ estudando a incidência de trombose venosa em pacientes submetidos a amputações maiores bilaterais em até 30 dias de pós-operatório, encontraram 4 casos de TVP e 4 casos de EP. Foram realizadas; no período do estudo, 27 amputações bilaterais; apenas 11 casos que apresentavam suspeita clínica de TVP foram estudados no pós-operatório e em nenhum caso foi realizado ecoDoppler no período pré-operatório.

Através de eco-Doppler realizado no $15^{\circ}$ pós-operatório, Burke et al. ${ }^{63}$ observaram presença de trombose venosa profunda do membro em 4 pacientes de 8 pacientes submetidos à amputação transtibial.

Frost et al. ${ }^{64}$ consideram o eco-Doppler como exame de escolha no diagnóstico da TVP proximal em doentes amputados. 
Chong et al. ${ }^{65}$ relataram 2 casos TVP no coto de amputação: um, 3 meses após o procedimento cirúrgico, e o outro, alguns anos após, ambos já reabilitados com prótese e deambulando.

Como podemos observar pelo exposto sobre a literatura, os estudos sobre a incidência de TVP e EP em pacientes submetidos à amputação cirúrgica das extremidades inferiores são controversos. Em grande parte dos trabalhos, são analisados pequenos números de casos com conclusões muitas vezes pouco confiáveis e não há nenhum estudo com casuística adequada especificando o período de pós-operatório necessário de investigação para avaliar o TEV. 


\section{OBJETIVOS}


Este estudo tem como finalidade avaliar primariamente a incidência de trombose venosa profunda no coto de amputação em pacientes com doença arterial oclusiva crônica periférica submetidos a amputações maiores do membro inferior, através do emprego de eco-Doppler, e secundariamente, a relação entre TVP, comorbidades e mortalidade operatória, no pós-operatório recente, utilizando método clínico, prospectivo, descritivo e não randomizado. 
3. MÉTODO 
Este é um estudo clínico prospectivo, consecutivo, não randomizado realizado no Hospital das Clínicas da Faculdade de Medicina da Universidade de São Paulo, no período de setembro de 2004 a março de 2006, com pacientes do Serviço de Cirurgia Vascular internados eletivamente ou no Pronto Socorro.

Esta pesquisa foi aprovada pelo Departamento de Cirurgia e pela Comissão de Ética e Pesquisa do Hospital das Clínicas da FMUSP (anexo A).

Todos os pacientes inclusos na pesquisa foram informados verbalmente sobre a importância do estudo, do que se tratava TVP e o objetivo. Além disso, leram o explicativo do estudo e, concordando, assinaram o termo de consentimento informado (anexo B).

\subsection{Critérios de Inclusão}

Foram critérios de inclusão: pacientes com diagnóstico de doença arterial obstrutiva crônica, que na admissão hospitalar apresentavam ausência de pulsos palpáveis no membro acometido, ou pacientes revascularizados previamente com pulsos palpáveis e que iriam ser submetidos à amputação maior de membro inferior, ou seja, amputação transtibial, transfemoral e desarticulação de joelho.

\subsection{Critérios de não Inclusão}

Não foram incluídos, no estudo, pacientes que sabidamente apresentavam ou tinham história confirmada de TVP no membro inferior que iria ser submetido à amputação ou no membro contralateral, pacientes que faziam uso de qualquer tipo de 
anticoagulantes e pacientes que não queriam fazer parte do estudo ou cujos familiares não concordaram, não assinando o termo de consentimento informado.

\subsection{Critérios de exclusão}

Foram excluídos do estudo pacientes que apresentaram trombose venosa profunda do membro inferior ao eco-Doppler no período pré-operatório, que apresentavam trombos no momento da secção da veia no intra-operatório, que não realizaram nenhum eco-Doppler no pós-operatório, seja por óbito ou perda de seguimento, uso de qualquer tipo de profilaxia com anticoagulantes e que apresentaram trombose venosa profunda no membro contralateral no período pósoperatório.

\subsection{Variáveis Pré-operatórias}

Foram avaliadas as variáveis: idade, sexo, presença de doenças associadas (quadro 1) e outros fatores considerados de risco para TVP (quadro 2). Não foram realizados exames de rotina específicos para confirmar os dados de anamnese informados pelos pacientes. Os exames realizados pelos pacientes eram solicitados pelos médicos que o estavam atendendo conforme achassem necessários, não havendo interferência do pesquisador. Consideramos a presença de cardiopatia quando o paciente referia ter insuficiência cardíaca ou coronariopatia isquêmica (infarto agudo do miocárdio prévio, angina). Para a obesidade, perguntávamos sobre o peso e altura do paciente e realizávamos o cálculo de índice de massa corpórea e 
este sendo maior do que 30, o paciente era considerado obeso. A imobilização no leito foi considerada quando o paciente não deambulava há pelo menos 30 dias. Varizes de membros inferiores foram avaliadas através do exame físico. Através da anamnese e exame físico, avaliamos se o paciente tinha alguma seqüela de acidente vascular cerebral como paresia ou paralisia. Quanto ao tabagismo, consideramos somente os ativos, independente da quantidade de cigarros/dia.

Quadro 1. Doenças associadas.

Cardiopatia
Tabagismo
Hipertensão arterial sistêmica
Diabetes Mellitus
Insuficiência renal crônica

Quadro 2. Fatores de risco para TVP avaliados.

Obesidade
Imobilização
Varizes de membros inferiores
Sequiela de Acidente Vascular Cerebral
Uso de hormônio feminino
Neoplasias

Analisamos se a indicação da amputação de membro inferior era somente pela presença de isquemia ou se havia também a presença de processo infeccioso. 
Analisamos o nível de oclusão arterial ao exame físico através da palpação dos pulsos do membro inferior acometido, conforme quadro 3.

Quadro 3. Nível de oclusão conforme a palpação dos pulsos.

\begin{tabular}{|l|l|}
\hline Nível de oclusão & Local da ausência de pulso palpável \\
\hline Ilíaco-femoral & Prega inguinal \\
Fêmoro-poplíteo & Cavo poplíteo \\
Poplíteo-distal & Pé \\
\hline
\end{tabular}

Analisamos ainda se o paciente havia sido submetido à revascularização do membro acometido no período até 30 dias prévio à indicação de amputação e se havia sido submetido anteriormente à amputação maior do membro inferior contralateral.

\subsection{Variáveis cirúrgicas}

Quanto à indicação de amputação e o nível em que iria ser realizada, não houve interferência do pesquisador, sendo a mesma indicada por critérios da equipe cirúrgica. Analisamos o nível de amputação realizada (transtibial, desarticulação de joelho e transfemoral), técnica aberta ou fechada e se havia a presença de trombo no local da secção da veia no intra-operatório. 


\subsection{Variáveis pós-operatórias}

Analisamos a presença das seguintes complicações no coto de amputação conforme o apresentado no quadro 4.

Quadro 4. Complicações no coto de amputação.

\begin{tabular}{|l|}
\hline Trombose venosa profunda \\
Necrose \\
Mista (infecção + necrose) \\
\hline Sinais e sintomas de TVP \\
\hline Perda de nível \\
\hline
\end{tabular}

No caso dos sinais de TVP no coto de amputação, consideramos como presentes quando os pacientes apresentavam aumento do volume do coto de amputação, empastamento da musculatura, aumento da circulação superficial e dor no trajeto vascular, exame clínico realizado sempre pelo pesquisador. Estes achados eram comparados, posteriormente, com o resultado do eco-Doppler colorido.

Analisamos a presença de outras complicações pós-operatórias conforme quadro 5. Se houvesse suspeita clínica de embolia pulmonar sintomática, o paciente seria submetido à angiotomografia computadorizada com protocolo para EP. A causa 
de óbito era informada pela equipe que estava cuidando do paciente e, em causas indeterminadas clinicamente, a causa mortis foi considerada a relatada pela necropsia.

Quadro 5. Outras complicações pós-operatórias.

\begin{tabular}{|l|} 
Clínicas \\
Embolia pulmonar sintomática \\
Óbito
\end{tabular}

\subsection{Eco-Doppler}

O eco-Doppler foi realizado no período pré-operatório e em 2 momentos no período pós-operatório, do $3^{\mathrm{o}}$ ao $15^{\mathrm{o}}$ dia e do $16^{\mathrm{o}}$ ao $33^{\mathrm{o}}$ dia, quando aconteceu o retorno dos doentes ao ambulatório e, antecipado, quando houvesse suspeita clínica de TVP.

Esses exames foram realizados por médico radiologista do Serviço de Radiologia do HCFMUSP no setor do Pronto Socorro, com o aparelho Logiq 5 (GE Healthcare, Milwaukee, WI, EUA). O paciente era posicionado em decúbito dorsal horizontal, e era utilizado transdutor multifrequencial linear de 7,5 MHZ. Foram estudadas as veias poplíteas, femorais (superficial e comum), ${ }^{66}$ femorais profundas e croças das safenas magnas de ambos os membros inferiores. O exame foi realizado, utilizando-se o modo B para avaliação da presença de material intraluminal e compressibilidade do vaso estudado. O modo colorido foi utilizado para avaliação da presença de fluxo e o modo espectral para avaliação da fasicidade respiratória e do padrão da curva da onda. ${ }^{22,26,67}$ Os exames não incluíram como rotina a avaliação 
das veias ilíacas, porém o exame seria realizado se houvesse alguma alteração do fluxo ou da fasicidade respiratória na veia femoral comum, que pudesse representar suspeita de oclusão ou compressão extrínseca da veia ilíaca, conforme descrito por Marz. ${ }^{68} \mathrm{O}$ exame foi considerado positivo para TVP quando havia presença de trombo com extensão maior que 5 centímetros e alteração do fluxo venoso. ${ }^{69}$

\subsection{Tratamento}

No acompanhamento pós-operatório, não havia interferência do pesquisador na forma como era realizado o tratamento das complicações, sejam elas clínicas ou no coto de amputação. Havia interferência do pesquisador para as datas de retornos pré-determinadas para realização do eco-Doppler e da antecipação da realização do mesmo quando havia suspeita clínica de TVP. Quando diagnosticada a TVP e o paciente estava hospitalizado, a equipe que estava cuidando do mesmo era comunicada e iniciado o tratamento de acordo com a preferência da mesma, HNF ou HBPM e posterior anticoagulação oral (Warfarina). Quando o diagnóstico foi em regime ambulatorial, o pesquisador comunicava a equipe do paciente e iniciava o tratamento com HBPM e anticoagulação oral. Todos os pacientes com TVP foram anticoagulados por um período de 6 meses, com retornos e controles laboratoriais periódicos. 


\subsection{Cálculo da Amostra}

Neste estudo, serão avaliados pacientes submetidos à amputação maior de membros inferiores e a chance de ocorrência de TVP. Em pesquisas anteriormente realizadas, observou-se que a proporção de TVP varia entre $10 \%$ e $30 \%$ com valor aproximado de $20 \%$. Entretanto, esta estimativa pontual é insuficiente, pois a probabilidade de que a estimativa adotada venha a coincidir com o verdadeiro valor do parâmetro é, em geral, nula ou praticamente nula. Isso decorre de os estimadores serem variáveis aleatórias, muitas vezes contínuas, logo, as estimativas obtidas quase certamente serão distintas do valor do parâmetro estudado. Ou seja, é quase certo que estejamos cometendo um erro de estimação, quando procedemos à estimativa por ponto de um parâmetro populacional. Devido a esse fato, surge a idéia de se construir um intervalo em torno da estimativa por ponto, de modo que esse intervalo tenha uma probabilidade conhecida de conter o verdadeiro valor do parâmetro. Essa é a idéia da estimação por intervalo. Ao intervalo que, com probabilidade conhecida, deverá conter o valor real do parâmetro, chamaremos de Intervalo de Confiança para esse parâmetro. À probabilidade, que designamos de (1- a), de que um intervalo de confiança contenha o valor do parâmetro, chamaremos de nível de confiança do respectivo intervalo.

Pela teoria da Inferência Estatística temos que o intervalo de confiança em nível de (1- a )\% para a proporção populacional é dado por:

$\mathrm{p}^{\prime} \pm \mathrm{z}$ a $/ 2\left(\left(\mathrm{p}^{\prime}\left(1-\mathrm{p}^{\prime}\right)\right) / \mathrm{n}\right) 1 / 2$ 
onde:

p' é a estimativa pontual da proporção populacional.

\section{N é o tamanho amostral}

z a /2 é o percentual a /2 da distribuição Gaussiana (ou Normal).

Isto significa que:

$\mathrm{P}\left(\mathrm{p}^{\prime}-\mathrm{z}\right.$ a $/ 2\left(\left(\mathrm{p}^{\prime}\left(1-\mathrm{p}^{\prime}\right)\right) / \mathrm{n}\right) 1 / 2 \leq \mathrm{p} \leq \mathrm{p}^{\prime}+\mathrm{z}$ a $\left./ 2\left(\left(\mathrm{p}^{\prime}\left(1-\mathrm{p}^{\prime}\right)\right) / \mathrm{n}\right) 1 / 2\right)=(1-\mathrm{a})$

Temos que o Intervalo de Confiança para a proporção populacional tem a semi-amplitude dada por:

$$
e=\mathrm{z} \text { a } / 2\left(\left(\mathrm{p}^{\prime}\left(1-\mathrm{p}^{\prime}\right)\right) / \mathrm{n}\right) 1 / 2
$$

assim, quando fixamos a e n determinamos o valor de $e$. Podemos também fixar os valores de a e $e$, determinar o valor de n, que é o problema da determinação do tamanho da amostra necessária para se realizar a estimação por intervalo com confiança e precisão desejadas. Trabalhando matematicamente a expressão acima, obtemos:

$\mathrm{n}=(\mathrm{z}$ a $/ 2 / e) 2$ p'(1-p')

Assim, para este estudo, consideramos que a proporção de TVP em estudo na amostra a ser avaliada esteja próxima de $20 \%(0,20)$ e fixamos os valores de a e $e$ em: a $=0,05$ e $e=0,10$, de tal forma que:

$\mathrm{P}(0,10 \leq \mathrm{p} \leq 0,30)=0,95$

Assim, para a obtenção de uma estimativa da proporção de TVP e seu respectivo intervalo de confiança a 95\%, necessitamos de, no mínimo, 62 pacientes. 


\subsection{Análise estatística}

As incidências cumulativas de TVP e óbito foram determinadas pelo método de Kaplan-Meier. A análise univariada dos fatores de risco para TVP foi obtida pelo método de Log-Rank, enquanto a regressão de Cox foi aplicada para análise multivariada. Foi considerado um valor estatístico significativo $\mathrm{P}<0,05$. 
4. RESULTADOS 
No período estudado, foram realizadas 88 amputações em 82 pacientes com DAOP. Foram excluídos 26 pacientes segundo tabela 1.

Tabela 1: Causas de exclusão dos pacientes do estudo $(n=26)$.

\begin{tabular}{lc}
\hline \multicolumn{1}{c}{ CRITÉRIO DE EXCLUSÃO } & $\begin{array}{c}\mathrm{N}^{\text {o de }} \\
\text { pacientes }\end{array}$ \\
\hline Presença de TVP pré-operatória & 6 \\
TVP diagnosticada no intra-operatório & 2 \\
TVP contralateral no período pós-operatório & 2 \\
Eco-Doppler pós-operatório não realizado (falta de condições e/ou óbito) & 9 \\
Pacientes em uso de anticoagulante no pós-operatório & 4 \\
Complicação de coto de amputação com desarticulação coxofemoral & 1 \\
Perda de seguimento & 2 \\
\hline
\end{tabular}

n- número de pacientes

Foram estudadas 62 amputações em 56 pacientes, sendo que em três casos a amputação foi bilateral e em três outros casos houve a necessidade de nova amputação proximal após 30 dias da primeira cirurgia.

A média de idade dos doentes foi de 67,1 +/- 11,6 anos (43-88), mediana 65,5 e desses, $51,7 \%$ eram homens. As doenças associadas e prevalência estão listadas na tabela 2. Dos 56 pacientes estudados, 37 (66 \%) estavam incapacitados de deambular e $12(21,4 \%)$ pacientes eram tabagistas.

Tabela 2: Prevalência de Doenças Associadas $(\mathrm{n}=60)$.

\begin{tabular}{lcc}
\hline & $\mathrm{N}$ & $\%$ \\
\hline Hipertensão arterial sistêmica & 48 & 80 \\
Diabetes mellitus & 42 & 70 \\
Cardiopatias & 16 & 26,7 \\
Varizes de membros inferiores & 14 & 23,3 \\
Acidente vascular cerebral & 13 & 21,7 \\
Obesidade & 7 & 11,7 \\
\hline
\end{tabular}

$\mathrm{n}$ - número de pacientes 
Seis doentes apresentavam o membro contralateral já amputado (04 transtibial e 02 transfemoral) e 02 doentes foram submetidos à amputação transtibial bilateral no mesmo ato operatório. Em todos os casos, a indicação primária de amputação foi por isquemia crítica associada à necrose da extremidade. Em 76,7\% dos casos, havia ainda a presença de processo infeccioso associado. Do total de pacientes, $12,9 \%$ haviam sido submetidos a algum tipo de revascularização arterial no período prévio de 30 dias.

A localização da doença arterial obstrutiva nos membros amputados foi predominante no segmento femoropoplíteo, em $71 \%$ dos casos. Os segmentos iliacofemoral e infrapoplíteo apresentaram incidência de 14,5\%.

O nível de amputação mais freqüente foi o transtibial, em $58,1 \%$. O membro inferior esquerdo foi o sítio de amputação em 51,6\% dos casos. Nenhum paciente foi submetido à desarticulação de joelho ou a amputação pela técnica aberta.

As complicações clínicas ocorreram em $20 \%$ dos pacientes e foram as seguintes: Broncopneumonia (11.7\%), peritonite e infarto agudo do miocárdio (3.3\% cada) e septicemia (1,7\%). Houve 1 caso $(1,6 \%)$ de EP sintomática não fatal. A incidência de óbito, no período de 30 dias, foi de $9.7 \%$.

Complicações em coto de amputação ocorreram em 45,3\% das amputações e as causas estão listadas na tabela 3. Em cinco casos de amputação transtibial, houve necessidade de re-amputação proximal precoce (30 dias).

Tabela 3. Causas de complicação no coto e nível de amputação.

\begin{tabular}{lcccc}
\hline \multicolumn{1}{c}{ Nível } & Infecção & Isquemia & Infecção e isquemia & $\begin{array}{c}\text { Total de } \\
\text { amputações }\end{array}$ \\
\hline Transfemoral & 4 & 0 & 01 & 26 \\
Transtibial & 12 & 07 & 03 & 36 \\
Total & 16 & 07 & 04 & 62 \\
\hline
\end{tabular}


Foram diagnosticados $16(25,8 \%)$ casos de TVP do coto amputação no pósoperatório (tabela 4), sendo 12 (75\%) nos primeiros 15 dias e quatro, após esse período. Em três pacientes, ocorreu também TVP no membro contralateral. Dos 16 casos, $14(87,5 \%)$ foram diagnosticados em regime ambulatorial. A média de dias da realização do primeiro eco-Doppler pós-operatório foi 6,4 (3-14) e o segundo de 31,9 (25-64). Não houve nenhum caso de TVP ilíacofemoral.

Tabela 4. Segmento venoso acometido de TVP e nível de amputação realizado.

\begin{tabular}{cccc}
\hline Segmento venoso & Transfemoral & Transtibial & Total \\
\hline Femoral & 10 & 4 & 14 \\
Poplíteo & 0 & 1 & 1 \\
Femoropoplíteo & 0 & 1 & 1 \\
Total & 10 & 6 & 16 \\
\hline
\end{tabular}

A incidência cumulativa de TVP, no coto de amputação, foi de $24.4 \%$ em 30 dias e $28 \%$ até o período de 35 dias, limite do erro padrão (gráfico 1). 
Gráfico 1: Incidência cumulativa de trombose venosa de coto de amputação (KaplanMeier).

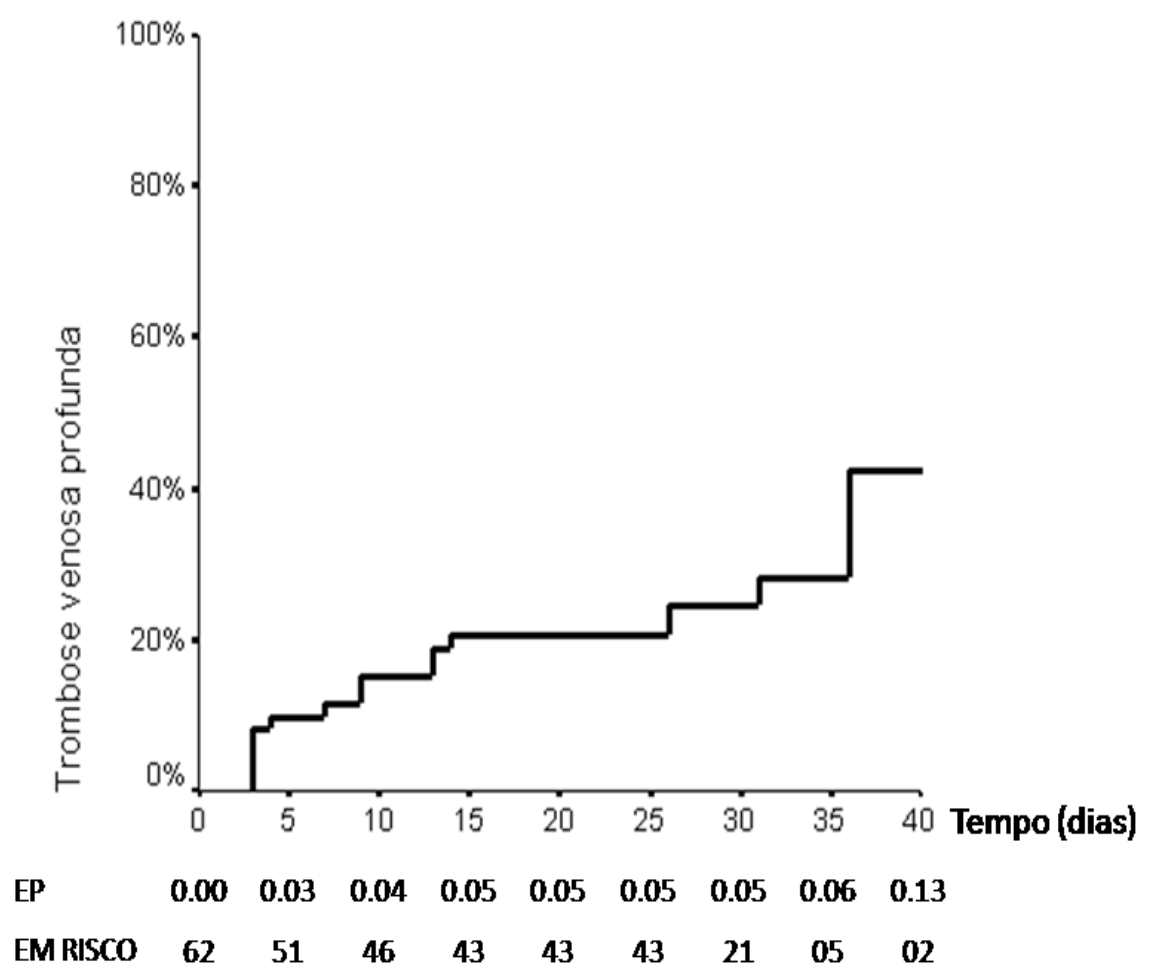

A avaliação clínica dos sinais e sintomas de TVP do coto de amputação apresentou sensibilidade de 56,2\%, especificidade de $82,6 \%$, valor preditivo negativo de $84,4 \%$ e valor preditivo positivo de $52,9 \%$.

A análise univariada (tabela 5) evidenciou diferença significativa na incidência de trombose venosa de coto quando comparados os níveis de amputação. As amputações transfemorais apresentaram incidência cumulativa de trombose venosa de $37,5 \%$, contra $21,2 \%$ nos pacientes submetidos à amputação transtibial até o $35^{\circ}$ dia de pós-operatório ( $\mathrm{p}=$ 0.04; gráfico 2).

A análise multivariada não mostrou associação entre fatores de risco e a ocorrência de TVP do membro amputado. 
Tabela 5: Análise univariável de fatores de risco para trombose venosa profunda de coto de amputação (Log Rank).

\begin{tabular}{|c|c|c|c|c|c|}
\hline FATOR & & $\mathrm{N}$ & TVP POR 100 INDIV / DIA & TAXA DE EVENTOS & $\mathrm{P}$ \\
\hline \multirow{2}{*}{ SEXO } & MASC & 31 & 1,506 & 1 & 0.13 \\
\hline & FEM & 31 & 0,725 & 0.48 & \\
\hline \multirow{2}{*}{$\begin{array}{l}\text { HIPERTENSÃO } \\
\text { ARTERIAL }\end{array}$} & SIM & 50 & 1,063 & 0.95 & 0.89 \\
\hline & NÃO & 12 & 1,115 & 1 & \\
\hline \multirow{2}{*}{ DIABETES MELLITUS } & SIM & 44 & 0,810 & 0.44 & 0.09 \\
\hline & NÃO & 18 & 1,837 & 1 & \\
\hline \multirow{2}{*}{ TABAGISMO } & SIM & 13 & 0,929 & 0.84 & 0.69 \\
\hline & NÃO & 49 & 1,112 & 1 & \\
\hline \multirow{2}{*}{ ACAMADO } & SIM & 39 & 0,819 & 0.53 & 0.20 \\
\hline & NÃO & 23 & 1,557 & 0 & \\
\hline \multirow{2}{*}{$\begin{array}{l}\text { NÍVEL } \\
\text { AMPUTAÇÃO }\end{array}$} & COXA & 26 & 1,792 & 1 & 0.044 \\
\hline & PERNA & 36 & 0,643 & 0.36 & \\
\hline \multirow{2}{*}{ IDADE } & $<70$ & 36 & 0,590 & 0.28 & 0.012 \\
\hline & $\geq 70$ & 26 & 2,105 & 1 & \\
\hline \multirow{2}{*}{ LADO } & ESQ & 32 & 0,960 & 0.81 & 0.55 \\
\hline & DIR & 30 & 1,180 & 1 & \\
\hline \multirow{2}{*}{ OBESIDADE } & SIM & 8 & 0,885 & 0.62 & 0.66 \\
\hline & NÃO & 54 & 1,419 & 1 & \\
\hline \multirow{2}{*}{$\begin{array}{c}\text { ISQUEMIA / } \\
\text { INFECÇÃO DO MEMBRO }\end{array}$} & SIM & 48 & 1,025 & 0.83 & 0.76 \\
\hline & NÃO & 14 & 1,242 & 1 & \\
\hline \multirow{2}{*}{ REAMPUTAÇÃO } & SIM & 5 & 2,273 & 1 & 0.66 \\
\hline & NÃO & 57 & 1,036 & 0.46 & \\
\hline \multirow{2}{*}{$\begin{array}{l}\text { AMPUTAÇÃO } \\
\text { CONTRALATERAL }\end{array}$} & SIM & 6 & 1,460 & 1 & 0.67 \\
\hline & NÃO & 56 & 1,033 & 0.71 & \\
\hline \multirow{2}{*}{ AVC } & SIM & 14 & 1,163 & 1 & 0.94 \\
\hline & NÃO & 48 & 1,045 & 0.90 & \\
\hline \multirow{2}{*}{ CARDIOPATIA } & SIM & 17 & 1,626 & 1 & 0.37 \\
\hline & NÃO & 45 & 0,890 & 0.55 & \\
\hline \multirow{2}{*}{ REVASCULARIZAÇÃO } & SIM & 8 & 1,031 & 0.96 & 0.99 \\
\hline & NÃO & 54 & 1,078 & 1 & \\
\hline \multirow{2}{*}{$\begin{array}{l}\text { COMPLICAÇÕES } \\
\text { DO COTO }\end{array}$} & SIM & 27 & 1,250 & 1 & 0.67 \\
\hline & NÃO & 35 & 0,939 & 0.75 & \\
\hline \multirow{2}{*}{$\begin{array}{l}\text { COMPLICAÇÕES } \\
\text { CLINICAS }\end{array}$} & SIM & 11 & 0,725 & 0.63 & 0.44 \\
\hline & NÃO & 51 & 1,151 & 1 & \\
\hline \multirow[t]{2}{*}{ ÓBITO } & SIM & 6 & 3,278 & 1 & 0.20 \\
\hline & NÃO & 56 & 0,978 & 0.3 & \\
\hline \multirow{2}{*}{ VARIZES } & SIM & 15 & 1,312 & 1 & 0.72 \\
\hline & NÃO & 47 & 0,990 & 0.75 & \\
\hline
\end{tabular}


Gráfico 2: Incidência cumulativa de trombose venosa de coto de amputação dividida pelo nível de amputação (Log-Rank).

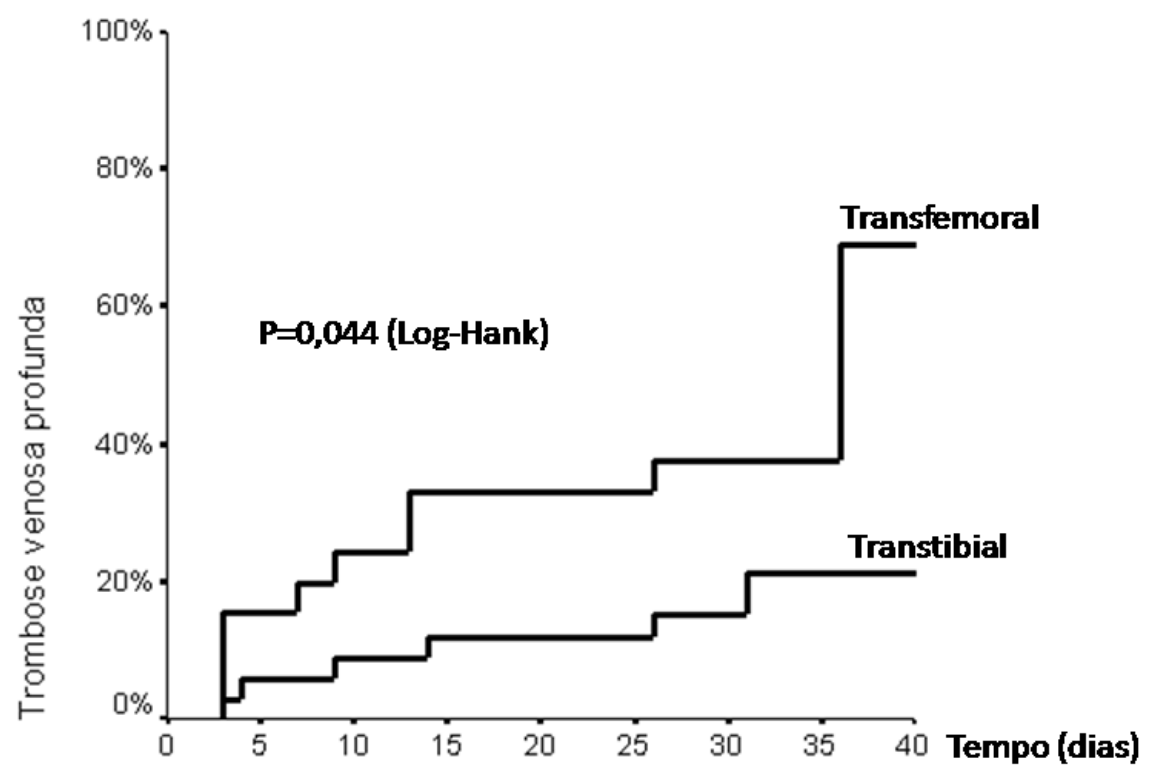

$\begin{array}{llccccccccc}\text { Transfemoral } & \text { EP } & 0.00 & 0.07 & 0.08 & 0.09 & 0.09 & 0.09 & 0.10 & 0.10 & 0.22 \\ & \text { EM RISCO } & 26 & 20 & 17 & 15 & 15 & 15 & 07 & 02 & 01 \\ & & & & & & & & & & \\ \text { Transtibial } & \text { EP } & 0.00 & 0.04 & 0.04 & 0.05 & 0.05 & 0.05 & 0.06 & 0.08 & 0.08 \\ & \text { EM RISCO } & 36 & 31 & 29 & 28 & 28 & 28 & 14 & 03 & 01\end{array}$

Pacientes com idade igual ou superior a 70 anos apresentaram risco de trombose de coto significativamente maior que os mais jovens no período de 35 dias (48.9 vs $16.8 \%$, respectivamente; $\mathrm{p}=0,012$ ), gráfico 3 . 
Gráfico 3: Incidência cumulativa de trombose venosa de coto de amputação dividida pela faixa etária (Log-Rank).

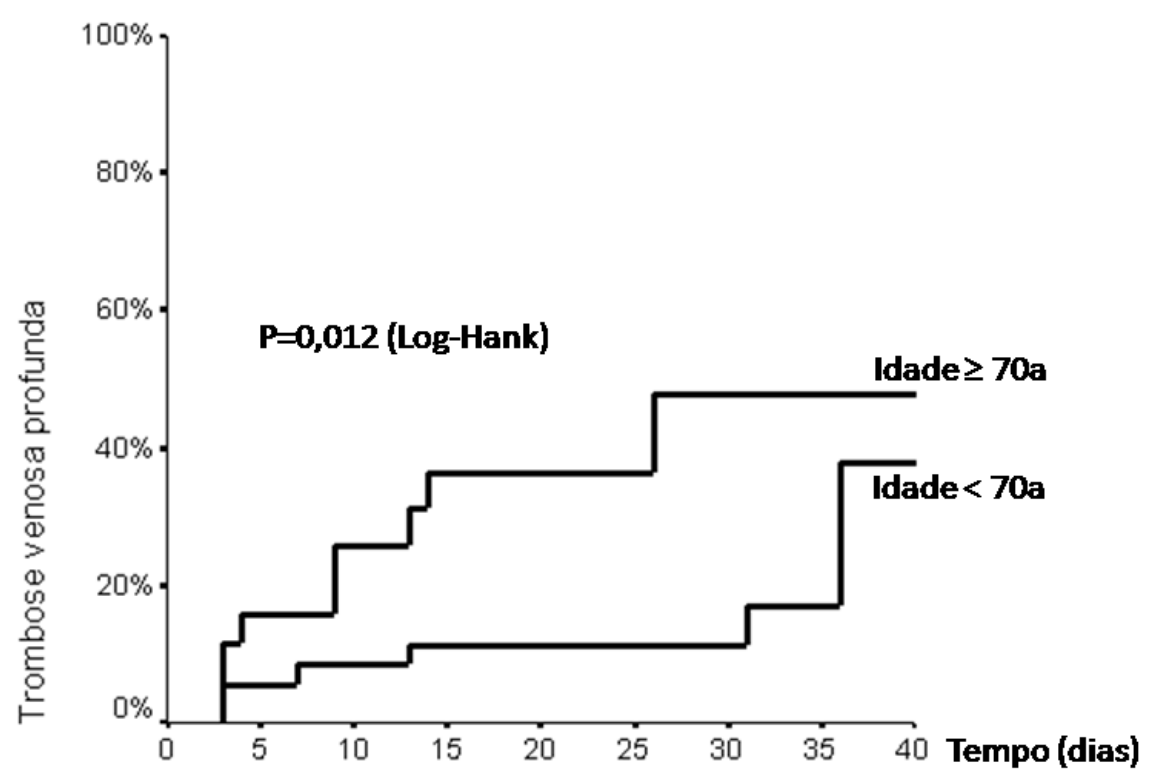

$\begin{array}{llccccccccc}\text { Idade } \geq 70 & \text { EP } & 0.00 & 0.07 & 0.09 & 0.10 & 0.10 & 0.10 & 0.11 & 0.11 & 0.11 \\ & \text { EM RISCO } & 36 & 18 & 14 & 12 & 12 & 12 & 05 & 01 & 01 \\ \text { Idade }<70 & \text { EP } & 0.00 & 0.03 & 0.04 & 0.05 & 0.05 & 0.05 & 0.05 & 0.07 & 0.18 \\ & \text { EM RISCO } & 26 & 33 & 32 & 31 & 31 & 31 & 16 & 04 & 01\end{array}$


5. DISCUSSÃO 
O número de pacientes inclusos na amostra mostrou-se suficiente de acordo com cálculo prévio. Esse número poderia ter sido maior se não tivéssemos estabelecido critérios bem definidos de não inclusão ou exclusão dos pacientes com TVP pré-operatória e trans-operatório, e pós-operatória no membro contralateral isoladamente, pois o objetivo do trabalho é estudar somente a incidência de TVP pós-operatória no membro amputado.

O eco-Doppler, hoje, é considerado padrão ouro para o diagnóstico de TVP. Devido às dificuldades técnicas de realizar preparo abdominal em todos os pacientes, não incluímos de rotina a avaliação do segmento venoso ilíaco. Optou-se por realizar o exame desse segmento na presença de alterações do fluxo na veia femoral como já descrito no método. Não houve a necessidade, em nosso estudo, da realização do eco-Doppler desse segmento, já que não foram encontradas tais alterações na veia femoral. Não realizamos pesquisa também para avaliação de TVP das veias da perna (distal) pois, segundo dados da literatura, o exame nesse segmento tem uma sensibilidade e especificidade menor para o diagnóstico de TVP em assintomáticos em relação ao segmento proximal. ${ }^{23,24}$ Além disso, como o objetivo era avaliar a incidência de TVP no coto de amputação no pós-operatório, não haveria tecnicamente condições de avaliação do sistema venoso do coto residual de perna, por isso optamos por avaliar a partir da veia poplítea. Para afastarmos qualquer dúvida se havia TVP no momento da cirurgia, os locais da secção venosa de todos os níveis eram observados quanto à presença de coágulos em seu interior. A maioria dos trombos originados nas veias das pernas apresenta resolução espontânea segundo Kearon ${ }^{12}$ e, segundo Labropoulos et al., ${ }^{36}$ independente de tratamento com 
anticoagulante ou expectante, as taxas de progressão do trombo para segmento proximal são equivalentes.

Trinta e oito por cento dos pacientes excluídos foram devido a diagnóstico de TVP pré ou intra-operatório, o que pode ser considerado um número relativamente elevado. Foram excluídos, ainda, 4 pacientes que realizaram profilaxia farmacológica enquanto estiveram internados na Unidade de Terapia Intensiva ( o protocolo da unidade recomenda o uso de profilaxia com HBPM ou HNF em todos os pacientes internados quando não há contra-indicação).

Esses dados permitem deduzir a gravidade dos pacientes da amostra com isquemia crítica, restritos ao leito e, com certeza, em estado francamente prótrombótico. A maior parte dos nossos pacientes foi proveniente do Pronto Socorro do HCFMUSP.

A média de idade foi relativamente elevada, provavelmente, por serem estudados pacientes somente com DAOP. Se fossem incluídas no estudo amputações traumáticas e/ou pacientes somente com processos infecciosos, a média de idade seria menor, pois essas situações ocorrem geralmente em pessoas mais jovens.

As doenças associadas mais prevalentes em nosso estudo foram a hipertensão arterial sistêmica e o diabetes mellitus.. Esse fato também deve ter ocorrido devido aos pacientes estudados serem somente os portadores de DAOP.

A baixa incidência de cardiopatia se deve, provavelmente, ao fato de apenas termos nos baseado nos dados referidos pelo paciente na anamnese; não indicamos exames complementares para diagnósticos de cardiopatias assintomáticas, o que provavelmente elevaria o número de pacientes com essa condição. O tabagismo também teria maior freqüência se não considerássemos somente os fumantes ativos 
na época da internação. Esses dois fatores não apresentaram influência na incidência de TVP no membro amputado.

Quase 2/3 dos pacientes eram incapacitados de deambular e 1/4 eram portadores de varizes de membros inferiores; dois fatores que, além da obesidade, sabidamente influenciam na incidência pós-operatória de TVP ou em pacientes clínicos acamados. ${ }^{2,5,6,8-10}$ Porém neste estudo não influenciaram a ocorrência de TVP.

Todos os pacientes submetidos à amputação foram por isquemia crítica e, na sua maioria, associada a processo infeccioso. Khiabani et al. ${ }^{70}$ afirmam, em estudo, que a principal causa de edema unilateral em pacientes com isquemia crítica é a TVP, porque eles apresentam um baixo fluxo arterial onde a perfusão venosa é comprometida. Esses pacientes, em tese, também estariam mais predispostos a estado pró-trombótico, no entanto também não houve relação com a ocorrência de TVP.

A TVP também não está relacionada com a topografia da oclusão arterial e a realização de revascularização arterial prévia à amputação. A localização mais freqüente da lesão arterial obstrutiva foi a artéria femoral superficial, local de maior acometimento pela DAOP por ser segmento de condução com pouca rede colateral, sujeita a forças de torção, principalmente, no final do canal dos abdutores (local onde ocorrem as instabilidades de placa com oclusão da artéria). A nossa taxa de amputação, após revascularização, é semelhante ao encontrado na literatura, ${ }^{44,52,54,71}$ e o fato dos pacientes terem sido submetidos a duas operações não levou, em nossa casuística, a uma maior incidência de TVP.

Observamos 12,9\% de amputações bilaterais, dados concordantes com os encontrados na literatura, que refere valores entre 10,6 e $15 \% .{ }^{47,50}$ Não tivemos, em 
nosso trabalho, a presença de TVP em amputados bilateralmente. Esperávamos a ocorrência de algum caso, considerando que esses pacientes estiveram mais restritos ao leito e submetidos a amputações simultâneas em 2 casos. Nossos dados discordam dos de Zickler et al. ${ }^{62}$ que evidenciaram a presença de TVP em $11 \%$ dos pacientes amputados bilateralmente.

A complicação clínica mais freqüente foi a broncopneumonia. Provavelmente isso se deva ao fato de os pacientes estarem acamados, em sua grande maioria, no período pré e pós-operatório, aumentando as chances de acúmulo de secreções em vias aéreas e conseqüentemente infecções pulmonares. Nossos dados são discordantes da literatura em que há maior presença de complicações cardíacas no pós-operatório. ${ }^{43,45-47}$ Essas complicações clínicas, apesar de aumentarem o estado pró-trombótico dos pacientes, não influenciaram a taxa de TVP.

Em nossa casuística, houve apenas um caso $(1,6 \%)$ de EP não fatal sintomática, enquanto na literatura a freqüência é maior, variando entre 2,1 e $8 \%$. ${ }^{47,53}$ Acreditamos que a baixa incidência de EP sintomática em nosso estudo se deve à vigilância diagnóstica com o eco-Doppler seriado e tratamento adequado imediato da TVP, o que pode ter diminuído consideravelmente a incidência de EP sintomática. Não realizamos exames de rotina para pesquisa de EP assintomática. Quando se realizam exames diagnósticos para esse fim, a incidência de EP na literatura varia de 11 a $14,3 \% .{ }^{56,72} \mathrm{O}$ estudo de Williams et al. ${ }^{58}$ atribui um risco de EP quatro vezes maior para os amputados em nível transfemoral. Em nosso estudo, não tivemos nenhum caso de EP em amputação transfemoral, apenas 01 caso em amputação transtibial. O paciente já tinha o diagnóstico de TVP no coto de amputação e, mesmo 
na vigência de tratamento adequado com anticoagulantes, apresentou EP, sendo então submetido à implante de filtro de veia cava.

Na literatura, a mortalidade operatória variou de 0 a $37 \%{ }^{51,53,73,74}$ e, em nosso estudo, observamos uma mortalidade operatória de 9,7\%, dentro dessa faixa de variação. De acordo com a análise estatística univariada, também não encontramos relação entre TVP e óbito em nossa casuística. A taxa de mortalidade seria maior se não tivéssemos excluído do estudo pacientes que morreram e/ou não puderam realizar eco-Doppler no pós-operatório.

Apesar de um número maior de pacientes apresentarem obstrução da artéria femoral superficial, foram realizadas mais amputações transtibiais. Esse nível é mais propício ao paciente pela manutenção da articulação do joelho, conseqüentemente menor gasto energético para locomoção e é preferida à amputação de coxa, mesmo sabendo-se que há uma maior chance de complicações locais. Todos os casos reamputados em nível mais proximal ocorreram em amputações transtibiais 5/36 $(13,9 \%)$, consoantes com dados da literatura, onde encontramos, em média, $15 \%$ de perda do nível em amputações transtibiais. ${ }^{49,53}$ A perda do nível da amputação transtibial provavelmente ocorreu, em parte devido aos casos amputados em nível inferior à oclusão arterial (ausência de pulso poplíteo palpável), e por esta região estar mais próxima do processo infeccioso distal. Cerca de $76,7 \%$ dos pacientes apresentavam isquemia associada à infecção no período pré-operatório. Esse fato deve ter colaborado para que a maior causa de complicação no coto de amputação transtibial fosse a infecção (54,5\%). Apesar disso, essas complicações e reamputações não influenciaram quanto à presença de TVP na análise univariada (tabela5). 
Com relação ao tempo de diagnóstico, a grande maioria de nossos casos de TVP ocorreram na primeira quinzena de pós-operatório ( $75 \%$ dos casos) e a taxa cumulativa estimada pelo método de Kaplan-Meier é de $28 \%$ até 35 dias (gráfico 1). Segundo Kearon, ${ }^{12} 61 \%$ dos casos de TVP em pacientes cirúrgicos ocorrem na primeira semana, mas pode acontecer até meses após o procedimento. Essa incidência ocorre, provavelmente, por ser o período mais recente em relação ao trauma cirúrgico, conseqüentemente maior estado pró-trombótico, e o período em que o paciente menos se movimenta no leito com receio de sentir dor.

Ao analisarmos a incidência de TVP no membro amputado no estudo de Harper et al. ${ }^{55}(66,7 \%)$, notamos uma incidência muito superior à obtida em nosso estudo (19,3\%) no mesmo período de observação no pós-operatório. Esta diferença pode ter ocorrido porque Harper et al. utilizou metodologia diagnóstica distinta, mantendo no pós-operatório cateter no interior da veia safena magna para executar flebografia, recurso invasivo com potencial trombogênico.

Quando comparamos nossos resultados com trabalho de Lastória et al. ${ }^{61}$ observamos uma incidência menor $(10,7 \%)$, mas muito próxima dos nossos resultados, tendo-se em vista que nesse estudo, ao contrário do nosso, foi utilizada profilaxia medicamentosa.

$\mathrm{Na}$ literatura, independente da casuística e metodologia utilizadas, também há uma maior incidência de TVP na primeira quinzena. ${ }^{59-65}$ Em nosso estudo, a grande diferença em relação às demais publicações da literatura é que, na maioria (87,5\%) dos casos, o diagnóstico foi ambulatorial, enquanto nos outros trabalhos a observação se limita apenas ao período em que o paciente se encontra internado. $\mathrm{O}$ tempo em que foram realizados os exames com eco-Doppler também foi adequado, 
abrangendo o período inicial ao trauma cirúrgico e o período mais tardio. Pudemos provar que os pacientes amputados não estão em risco para desenvolvimento de TVP somente no período inicial do pós-operatório, mas também após alta hospitalar.

A suspeita clínica de TVP no coto de amputação baseada nos dados de exame clínico obteve uma baixa sensibilidade, porém com valor preditivo negativo de $84,4 \%$, o que infere na necessidade da realização de um método diagnóstico mais específico mesmo em pacientes sem suspeita clínica.

Evidenciamos que o tipo de amputação realizada mostrou relação com a incidência de TVP, sendo que as amputações transfemorais tiveram incidência cumulativa de $31,5 \%$ contra $21,2 \%$ das transtibiais, dados com diferença estatisticamente significativa (gráfico 2). Provavelmente a maior incidência de TVP em pacientes com amputação transfemoral deve-se ao fato da veia femoral ser segmento de condução, com poucas tributárias. Para se ter certeza de que a trombose não seria exclusivamente decorrente da ligadura venosa da veia femoral, determinouse, no projeto, que a presença de TVP seria considerada apenas quando houvesse trombo com extensão igual ou superior a 5 centímetros, com conseqüente necessidade de tratamento específico, conforme descrito por Maffei. ${ }^{69}$

É importante salientar que, em nosso material, sem exceção, os casos de TVP da veia femoral superficial acometeram toda sua extensão. Em trabalho de Makki et al., ${ }^{67}$ estudando a localização do trombo no sistema venoso profundo de pacientes com TVP, a trombose isolada da veia femoral superficial ocorreu em 22,3\% dos casos e a extensão para a veia femoral comum ou poplítea, em 56,9\%, sem se referir quais foram as indicações para realização do exame e nem fatores de risco. Em nossa casuística, encontramos 56,2\% dos casos de TVP isolados na veia femoral 
superficial, sem diferença significativa entre os níveis de amputação. Em 31,2\% dos casos, houve extensão da trombose para a veia femoral comum ou poplítea, sendo mais freqüente a extensão para veia femoral comum em amputações transfemorais.

Outro fator que se mostrou positivo e estatisticamente significativo para a presença de TVP foi idade igual ou superior a 70 anos. Como já relatamos anteriormente, na literatura, há uma maior incidência de TVP em pacientes cirúrgicos com idade superior a 40 anos. Nós pesquisamos por faixas etárias e evidenciamos esse fato. Supõe-se que isso deva ocorrer pela acentuada demora na recuperação ao trauma cirúrgico do paciente mais idoso.

Todos os outros fatores estudados não apresentaram relação com a incidência maior de TVP (tabela 5), contrastando com os dados encontrados na literatura em pacientes clínicos e cirúrgicos de maneira geral. $6,8,11,29,37,75-79$

O trabalho de Yager et al. ${ }^{10}$ evidenciou, como fatores de risco para TVP, a insuficiência venosa crônica e/ou história prévia de TVP e a presença de amputação prévia contralateral. A análise também incluiu os casos diagnosticados no período pré-operatório 6/9 (66,7\%) e no membro contralateral 4/9 (44,4\%). Trabalho esse, com metodologia distinta do nosso estudo em que consideramos apenas os casos de TVP pós-operatória, com critérios de não inclusão e exclusão bem definidos.

A análise multivariada não foi capaz de avaliar a associação dos diversos fatores com a presença de TVP no pós-operatório.

Em resumo, é importante salientar que a amostra estudada não difere das características de grupos de pacientes amputados da literatura, bem como as complicações no membro amputado. Através de metodologia rigorosa e bem préestabelecida, pudemos provar que a incidência de TVP no coto de amputação é 
significativa mesmo após alta hospitalar. Portanto, esses pacientes devem ser cuidadosamente acompanhados no período pós-operatório. 


\section{CONCLUSÕES}


A incidência de TVP no coto de amputação é elevada no pós-operatório precoce (35 dias), principalmente nas amputações transfemorais e/ou idade igual ou superior a 70 anos. Não houve relação entre presença das outras comorbidades estudadas e ocorrência de TVP pós-operatória. A presença de TVP não influenciou na mortalidade operatória. Todo paciente com DAOP, a ser submetido à grande amputação, deve ser considerado de alto risco para o desenvolvimento de TVP, mesmo após alta hospitalar. 
7. Anexos 
ANEXO A

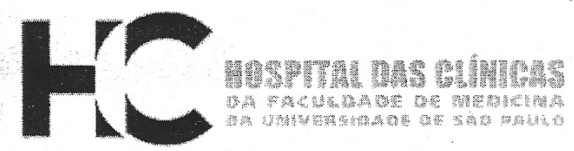

DIRETORIA CLINICA

Comissão de Ética para Análise de Projetos de Pesquisa APROVAÇÃO

A Comissão de Ética para Análise de Projetos de Pesquisa - CAPPesq da Diretoria Clínica do Hospital das Clínicas e da Faculdade de Medicina da Universidade de São Paulo, em sessão de 26.08.04, APROVOU o Protocolo de Pesquisa $n^{\circ}$ 698/04, intitulado: "Incidência de trombose yenosa profunda em pacientes com doença arterial oclusiva crônica submetidos a amputação do membro inferior" apresentado pelo Departamento de CIRURGIA, inclusive o Termo de Consentimento Livre e Esclarecido.

Pesquisador(a) Responsável: Prof. Dr. Calógero Presti

Pesquisador(a) Executante: Dr. Marcelo Fernando Matielo

CAPPesq, 26 de Agosto de 2004.

PROF. DR. EUCLIDES AYRES DE CASTILHO

Presidente da Comissão de Ética para Análise de Projetos de Pesquisa

OBSERVAÇÃO: Gabe ao pesquisador elaborar e apresentar à CAPPésq, os relatórios parciais e final sobre a pesquisa (Resolução do Conselho Nacional de Saúde $n^{\circ} 196$, de 10.10.1996, inciso IX.2, letra " $c$ ") 
HOSPITAL DAS CLÍNICAS

DA

Anexo B

FACULDADE DE MEDICINA DA UNIVERSIDADE DE SÃO PAULO

TERMO DE CONSENTIMENTO PÓS -INFORMAÇÃO

(Instruções para preenchimento no verso)

\section{I - DADOS DE IDENTIFICAÇÃO DO SUJEITO DA PESQUISA OU RESPONSÁVEL LEGAL}

1.NOME DO PACIENTE.

DOCUMENTO DE IDENTIDADE № :

SEXO : .M $\square \mathrm{F}$

DATA NASCIMENTO:

№

ENDEREÇO

APTO:

BAIRRO:

CIDADE

CEP: TELEFONE: DDD

2.RESPONSÁVEL

LEGAL

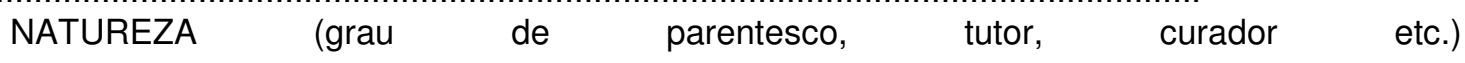

DOCUMENTO DE IDENTIDADE SEXO: $M \square \quad F$

DATA NASCIMENTO.: .....................

№

APTO:

BAIRRO:

CIDADE:

CEP:

TELEFONE:

DDD 


\section{II - DADOS SOBRE A PESQUISA CIENTÍFICA}

1. Título do PROTOCOLO DE PESQUiSA - avaliação da incidência de trombose venosa profunda no coto de amputação dos pacientes submetidos a amputações maiores de membros inferiores com doença arterial oclusiva, no pós-operatório recente

2. PESQUISADOR RESPONSÁVEL : CALÓGERO PRESTI

3. PESQUISADOR EXECUTANTE : MARCELO FERNANDO MATIELO

CARGO/FUNÇÃO:PÓS-GRADUANDO LATU SENSU 75362

INSCRIÇÃO CONSELHO REGIONAL №

UNIDADE DO HCFMUSP: DISCIPLINA DE CIRURGIA VASCULAR PERIFÉRICA DEPARTAMENTO DE CIRURGIA

3. AVALIAÇÃO DO RISCO DA PESQUISA:

SEM RISCO $\square$ X

RISCO BAIXO

(probabilidade de que o indivíduo sofra algum dano como consequência imediata ou tardia do estudo)

\section{III - REGISTRO DAS EXPLICAÇÕES DO PESQUISADOR AO PACIENTE OU SEU REPRESENTANTE LEGAL SOBRE A PESQUISA, CONSIGNANDO:}

- Este estudo irá avaliar a incidência (quantos casos acontecem) de trombose venosa profunda (que é a formação de coágulo nas veias que ficam dentro da perna, no meio da musculatura) em pacientes que tiveram a perna ou a coxa amputada.

- O paciente fará exame de eco-Doppler venoso (ultra-som vascular, um exame não agressivo; sem necessidade de punções venosas ou injeções de contraste), sem risco de vida, antes da operação de amputação. O exame será repetido antes da alta hospitalar e 30 dias depois da operação.

- O estudo permitirá verificar a formação desses coágulos, o mais cedo possível, para ser realizado tratamento mais adequado no sentido de tentar evitar a embolia pulmonar, que é a saída do coágulo da perna e indo parar no pulmão. 


\section{IV - ESCLARECIMENTOS DADOS PELO PESQUISADOR SOBRE GARANTIAS DO SUJEITO DA PESQUISA:}

- O paciente ou seu responsável têm direito a serem informados sobre suas dúvidas, os riscos e as vantagens do tratamento, a qualquer momento que julgarem necessário.

- O paciente ou seu responsável têm direito a retirar sua autorização para a realização do exame com eco-Doppler, mesmo que este já tenha se iniciado. Caso isto ocorra, ele continuará seu tratamento sem a realização do ultra-som neste mesmo hospital.

- Em nenhum momento o paciente terá seu nome ou dados pessoais divulgados pela equipe médica.

- Caso o paciente apresente complicações relacionadas ao tratamento, ele terá toda assistência médico-hospitalar por parte do Hospital das Clínicas da Faculdade de Medicina da Universidade de São Paulo.

\section{INFORMAÇÕES DE NOMES, ENDEREÇOS E TELEFONES DOS RESPONSÁVEIS PELO ACOMPANHAMENTO DA PESQUISA, PARA CONTATO EM CASO DE INTERCORRÊNCIAS CLÍNICAS E REAÇÕES ADVERSAS.}

Dr. Marcelo Fernando Matielo - Cremesp no 75362 End. Profissional: HOSPITAL DAS CLÍNICAS - Rua Enéas de Carvalho Aguiar, $255-6^{\circ}$ andar, bloco 5. Bairro Pinheiros CEP 05403-001 São Paulo - SP. Fone: 3069-6101. End. Comercial: Rua Dr. Neto de Araújo, 320, conj. 1007. Bairro Vila Mariana, CEP 04111-001 São Paulo - SP.TELEFONES: comercial: 5571-6696 celular: 9901-8550 bip: 5508-0737 código 4015972.

Dr.Calógero Presti - End. Profissional: HOSPITAL DAS CLÍNICAS - Rua Enéas de Carvalho Aguiar, 255 - $6^{\circ}$ andar, bloco 5. Bairro Pinheiros CEP 05403-001 São Paulo - SP TELEFONES: consultório:287-5281 celular: 9982-3717 HC: 30696101

\section{OBSERVAC̄ÕES COMPLEMENTARES:}

O PACIENTE E SEU RESPONSÁVEL TÊM À SUA DISPOSIÇÃO UM FOLHETO EXPLICATIVO EM LINGUAGEM SIMPLES, RESPONDENDO ÀS DÚVIDAS MAIS FREQÜENTES SOBRE O QUE É TROMBOSE VENOSA PROFUNDA, SUAS COMPLICAÇÕES E A IMPORTÂNCIA DE SEU DIAGNÓSTICO PRECOCE.

\section{VII - CONSENTIMENTO PÓS-ESCLARECIDO}

Declaro que, após convenientemente esclarecido pelo pesquisador e ter entendido o que me foi explicado, consinto em participar do presente Protocolo de Pesquisa.

São Paulo, de de 20 
8. REFERÊNCIAS 
1. Kakkar VV, Stringer MD. Prophylaxis of venous thromboembolism. World J. Surg. 1990; 14(5):670-8.

2. Geerts WH, Heit JA, Clagett GP, Pineo GF, Colwell CW, Anderson FA Jr, Wheeler HB. Prevention of venous thromboembolism. Chest 2001; 119(1Suppl):132S-175S.

3. De Castro-Silva M. Venous thromboembolism in the State of Minas Gerais and its projection to Brazil: study based in 2,331,353 hospitalisations. Int Angiol 1997; 16(3): 193-6.

4. Stein PD, Patel KC, Kalra NK, El Baage TY, Savarapu P, Silbergleit A, Check FE. Deep venous thrombosis in a general hospital. Chest 2002; 122(3): 960-2.

5. Anderson FA Jr, Wheeler HB. Physician practices in the management of venous thromboembolism: a comunity-wide survey. J Vasc Surg. 1992; 16(5): 707-14.

6. Heit JA, O'Faloon WM, Petterson TM, Lohse CM, Silverstein MD, Mohr DN, Melton LJ 3rd. Relative impact of risk factors for deep vein thrombosis and pulmonary embolism: a population based study. Arch Intern Med. 2002; 162(11): 1245-48.

7. White RH, Zhou H, Romano PS. Incidence of idiopathic deep venous thrombosis and secondary thromboembolism among ethnic groups in California. Ann Intern Med. 1998; 128(9): 737-40.

8. Weinmann EE, Salzman EW. Deep-vein thrombosis. N Engl J Med 1994; 331(24):1630-41 
9. Kakkar VV, Howe CT, Nicolaides AN, Renney JT, Clarke MB. Deep vein thrombosis of the leg. Is there a "high risk" group? Am J Surg 1970; 120(4):527-30.

10. Yeager RA, Moneta GL, Edwards JM, Taylor LM Jr, McConnell DB, Porter JM. Deep vein thrombosis associated with lower extremity amputation. J Vasc Surg 1995; 22(5):612-5.

11. Fowkes FJ, Price JF, Fowkes FG. Incidence of diagnosed deep vein thrombosis in the general population: systematic review. Eur $\mathrm{J}$ Vasc Endovasc Surg 2003; 25(1): 1-5.

12. Kearon C. Natural history of venous thromboembolism. Circulation 2003; 107(23 Suppl 1): I22-30

13. Birdwell B. Recent clinical trials in the diagnosis of deep-vein thrombosis. Curr Opin Hematol 1999; 6(5): 275-9.

14. Ramzi DW, Leeper KV. DVT and pulmonary embolism: Part I. Diagnosis. Am Fam Physician 2004; 69(12): 2829-36.

15. Morimoto Y, Sugimoto T, Mukai T, Okita Y, Okada M. Diagnosis of deep vein thrombosis using platelet scintigraphy. Ann Thorac Cardiovasc Surg 2001; 7(3):138-42.

16. Lennox AF, Delis KT, Serunkuma S, Zarka ZA, Daskalopoulou SE, Nicolaides AN. Combination of a clinical risk assessment score and rapid whole blood D-dimer testing in the diagnosis of deep vein thrombosis in symptomatic patients. J Vasc Surg 1999; 30(5): 794-803. 
17. Kalodiki E, Calahoras LS, Delis KT, Zouzias CP, Nicolaides AN. Air plethysmography: the answer in detecting past deep venous thrombosis. J Vasc Surg 2001; 33(4):715-20.

18. Rosen CL, Tracy JA. The diagnosis of lower extremity deep venous thrombosis. Emerg Med Clin North Am 2001; 19(4): 895-912.

19. Fraser DG, Moody AR, Morgan PS, Martel AL, Davidson I. Diagnosis of lower-limb deep venous thrombosis: a prospective blinded study of magnetic resonance direct thrombus imaging. Ann Intern Med 2002;136(2): 89-98.

20. Patel S, Kazerooni EA. Helical CT for the evoluation of acute pulmonary embolism. AJR Am J Roentgenol 2005; 185(1): 135-149.

21. Meissner MH. Duplex follow-up of patients with DVT: does it have clinical significance? Semin Vasc Surg 2001; 14(3):215-21.

22. Zierler BK. Screening for acute DVT: optimal utilization of the vascular laboratory. Semin Vasc Surg 2001; 14(3):206-14.

23. Mattos MA, Londrey GL, Leutz DW, Hodgson KJ, Ramsey DE, Barkmeier LD, Stauffer ES, Spadone DP, Sumner DS. Color-flow duplex scanning for the surveillance and diagnosis of acute deep venous thrombosis. J Vasc Surg $1992 ; 15(2): 366-76$.

24. Elias A, Cadene A, Elias M, Puget J, Tricoire JL, Colin C, Lefebvre D, Rousseau H, Joffre F. Extended lower limb venous ultrasound for the diagnosis of proximal and distal vein thrombosis in asymptomatic patients after total hip replacement. Eur J Vasc Endovasc Surg 2004; 27(4): 438-44. 
25. Lohr JM, Hasselfeld KA, Byrne MP, Deshmukh RM, Cranley JJ. Does the assymptomatic limb harbor deep venous thrombosis? Am J Surg 1994; $168(2): 184-7$.

26. Zierler BK. Ultrasonography and diagnosis of venous thromboembolism. Circulation 2004; 109(12 Suppl 1): I9-14.

27. Ramzi DW, Leeper KV. DVT and pulmonary embolism: part II. Treatment and prevention. Am Fam Physician 2004; 69(12): 2841-8.

28. Kelly J, Rudd A, Lewis RR, Hunt BJ. Screening for subclinical deep-vein thrombosis. Q J M 2001; 94(10): 511-19.

29. Hansson PO, Sörbo J, Eriksson H. Recurrent venous thromboembolism after deep vein thrombosis: incidence and risk factors. Arch Intern Med 2000; 160(6): 769-74.

30. Kearon C, Gent M, Hirsh J, Weitz J, Kovacs MJ, Anderson DR, Turpie AG, Green D, Ginsberg JS, Wells P, MacKinnon B, Julian JA. A comparison of three months of anticoagulation with extended anticoagulation for a first episode of idiopathic venous thromboembolism. N Engl J Med 1999; 340(12): $901-7$.

31. Research Committee of The British Thoracic Society. Optimum duration of anticoagulation for deep-vein thrombosis and pulmonary embolism. Lancet 1992; 340: 873-76.

32. Heit JA, Mohr DN, Silverstein MD, Petterson TM, O'Fallon WM, Melton LJ 3rd. Predictors of recurrence after deep vein thrombosis and pulmonary embolism: a population-based cohort study. Arch Intern Med 2000; 160(6): 761-8. 
33. Schulman S, Rhedin AS, Lindmarker P, Carlsson A, Lärfars G, Nicol P, Loogna E, Svensson E, Ljungberg B, Walter H. A comparison of six weeks with six months of oral anticoagulation therapy after a first episode of venous thromboembolism. Duration of anticoagulation trial study group. $\mathrm{N}$ Engl J Med 1995; 332(25): 1661-5.

34. Turton EP, Coughlin PA, Berridge DC, Mercer KG. A survey of deep venous thrombosis management by consultant vascular surgeons in the United Kingdom and Ireland. Eur J Vasc Endovasc Surg 2001; 21(6): 55863.

35. Lagerstedt CI, Olsson CG, Fagher BO, Öqvist BW, Albrechtsson U. Need for long-term anticoagulant treatment in symptomatic calf-vein thrombosis. Lancet 1985; 2(8454): 515-8

36. Labropoulos N, Kang SS, Mansour MA, Giannoukas AD, Moutzouros V, Baker WH. Early thrombus remodelling of isolated calf deep vein thrombosis. Eur J Vasc Endovasc Surg 2002; 23(4): 344-8.

37. Pinede L, Ninet J, Duhaut P, Chabaud S, Demolombe-Rague S, Durieu I, Nony P, Sanson C, Boissel JP; Investigators of the "Durée Optimale du Traitement AntiVitamines K" (DOTAVK) Study. Comparison of 3 and 6 months of oral anticoagulant therapy after a first episode of proximal deep vein thrombosis or pulmonary embolism and comparison of 6 and 12 weeks of therapy after isolated calf deep vein thrombosis. Circulation 2001; 103(20): 2453-60.

38. Bates SM, Ginsberg JS. Clinical practice. Treatment of deep-vein thrombosis. N Engl J Med 2004; 351(3): 268-77. 
39. Weitz JI, Byrne J, Clagett GP, Farkouh ME, Porter JM, Sackett D, Strandness DE Jr, Taylor LM. Diagnosis and treatment of chronic arterial insufficiency of the lower extremities: a critical review. Circulation 1996; 94(11): 3026-49.

40. TransAtlantic Inter-Society Consensus - Management of peripheral arterial disease. Epidemiology, natural history, risk factors. J Vasc Surg. 2000;31(1):S5-34.

41. Kacy SS, Wolma FJ, Flye MW. Factors affecting the results of below-knee amputation in patients with and without diabetes. Surg Gynaecol Obstet $1982 ; 155(4): 513-18$

42. Spichler D, Miranda F Jr, Spichler ES, Franco LJ. Amputações maiores de membros inferiores por doença arterial periférica e diabete melito no município do Rio de Janeiro. J Vasc Br 2002; 3(2):111-122.

43. de Virgílio C, Toosie K, Lewis RJ, Stabile BE, Baker JD, White R, Donayre CE, Ephraim L. Cardiac morbidity and operative mortality following lowerextremity amputation: the significance of multiple Eagle criteria. Ann Vasc Surg 1999; 13(2): 204-8.

44. Dawson I, Keller BP, Brand R, Pesch-Batenburg J, Hajo van Bockel J. Late outcomes of limb loss after failed infrainguinal bypass. J Vasc Surg 1995; 21(4): 613-22.

45. Abou-Zamzam AM Jr, Teruya TH, Killeen JD, Ballard JL. Major lower extremity amputation in an academic vascular center. Ann Vasc Surg 2003; 17(1):86-90. 
46. Dossa CD, Shepard AD, Amos AM, Kupin WL, Reddy DJ, Elliot JP, Wilczwski JM, Enrst CB. Results of lower extremity amputations in patients with end-stage renal disease. J Vasc Surg 1994; 20(1): 14-9.

47. Nehler MR, Coll JR, Hiatt WR, Regensteiner JG, Schnickel GT, Klenke WA, Strecker PK, Anderson MW, Jones DN, Whitehill TA, Moskowitz S, Krupski WC. Functional outcome in a contemporary series of major lower extremity amputations. J Vasc Surg 2003; 38(1): 7-14.

48. Dwars BJ, van den Broek TA, Rauwerda JA, Bakker FC. Criteria for reliable selection of the lowest level of amputation in peripheral vascular disease. J Vasc Surg 1992; 15(3): 536-42.

49. Dormandy J, Belcher G, Broos P, Eikelboom B, Laszlo G, Konrad P, Moggi L, Mueller U. Prospective study of 713 below-knee amputations for ischaemia and the effect of a prostacyclin analogue on healing. Hawaii study group. Br J Surg 1994; 81(1): 33-7.

50. Nicholas GG, Myers JL, DeMuth WE Jr. The role of vascular laboratory criteria in the selection of patients for lower extremity amputation. Ann Surg 1982; 195(4): 469-73.

51. Schina MJ Jr, Atnip RG, Healy DA, Thiele BL. Relative risks of limb revascularization and amputation in the modern era. Cardiovasc Surg 1994; 2(6): 754-9.

52. Kazmers M, Satiani B, Evans WE. Amputation level following unsuccessful distal limb salvage operations. Surgery 1980; 87(6): 683-7.

53. Roon AJ, Moore WS, Goldstone J. Below-knee amputation: a modern approach. Am J Surg 1977; 134(1): 153-8. 
54. Huston CC, Bivins BA, Enrst CB, Griffen WO Jr. Morbid implications of above-knee amputations. Report of a series and review of the literature. Arch Surg 1980; 115(2): 165-7.

55. Harper DR, Dhall DP, Wooddruff PW. Prophylaxis in iliofemoral venous thrombosis. The major amputee as a clinical research model. Br J Surg 1973; 60(10):831.

56. Williams JW, Britt LG, Sherman RT. Pulmonary embolism associated with surgically proved deep venous thrombosis. Am J Surg 1975; 129(5): 500-2.

57. Barnes RW. Prospective screening for deep vein thrombosis in high risk patients. Am J Surg 1977; 134(2):187-90.

58. Williams JW, Eikman EA, Greenberg SH, Hewitt JC, Lopez-Cuenca E, Jones GP, Madden JA. Failure of low dose heparin to prevent pulmonary embolism after hip surgery or above the knee amputation. Ann Surg 1978; 188(4): $468-74$.

59. Fletcher JP, Batiste P. Incidence of deep vein thrombosis following vascular surgery. Int Angiol 1997; 16(1):65-8.

60. Muraco BN, Guimarães PCM, Molnar L, Fortunato F, Strefezza E, Kalume C, Langer B, Benabou J. Ocorrência de trombose venosa em coto de amputação. Cir. Vasc. \& Angiol 1993; 9(3supl):26.

61. Lastória S, Rollo HA, Yoshida WB, Giannini M, Moura R, Maffei FH. Prophylaxis of deep-vein thrombosis after lower extremity amputation: comparison of low molecular weight heparin with unfractionated heparin. Acta Cir Bras 2006; 21(3): 184-6. 
62. Zickler RW, Gahtan V, Matsumoto T, Kerstein MD. Deep venous thrombosis and pulmonary embolism in bilateral lower extremity amputee patients. Arch Phys Med Rehabil 1999; 80(5):509-11.

63. Burke B, Kumar R, Vickers V, Grant E, Scremin E. Deep vein thrombosis after lower limb amputation. Am J Phys Med Rehabil 2000; 79(2):145-9.

64. Frost FS, Kelly CM, McCarthy W. High resolution real-time ultrasound for the diagnosis of venous thrombosis in the rehabilitation setting. Am J Phys Med Rehabil 1991; 70(1):3-4.

65. Chong DK, Panju A. Deep venous thrombosis as a cause of stump swelling in two lower extremity amputee patients. Arch Phys Med Rehabil 1993; 74(9):1002-3.

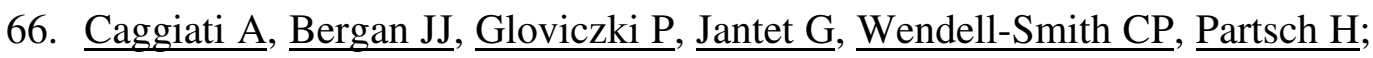
International Interdisciplinary Consensus Committee on Venous Anatomical Terminology. Nomenclature of the veins of the lower limbs: an international interdisciplinary consensus statement. J Vasc Surg 2002; 36(2): 416-22.

67. Maki DD, Kumar N, Ngyuen B, Langer JE, Millar WT Jr; Gefter WB. Distribution of thrombi in acute lower extremity deep venous thrombosis: implications for sonography and CT and MR venography. AJR Am J Roentgenol 2000; 175(5):1299-1301.

68. Marz BA. Avaliação da trombose venosa aguda com Duplex. In Strandness DE Jr. Doppler colorido nas doenças vasculares. $3^{\circ}$ ed. Rio de Janeiro: Guanabara-Koogan; 2002. 345-54.

69. Maffei FHA. Trombose venosa profunda dos membros inferiores: incidência, patologia, fisiopatologia e diagnóstico. In Maffei FHA, Lastória 
S, Yoshida WB, Rollo HA. Doenças Vasculares Periféricas. $2^{\circ}$ ed. Rio de Janeiro: Medsi; 1995. 841-62.

70. Khiabani HZ, Anvar MD, Straden E, Slagsvold CE, Kroese AJ. Oedema in the lower limb of patients with chronic critical limb ischaemia (CLI). Eur J Vasc Endovasc Surg 1999; 17(5): 419-23.

71. Whittaker L, Wijesinghe LD, Berridge DC, Scott DJ. Do patients with critical limb ischaemia undergo multiple amputations after infrainguinal bypass surgery? Eur J Vasc Endovasc Surg 2001; 21(5): 427-31.

72. Williams JW, Britt LG, Eades T, Sherman RT. Pulmonar embolism after amputation of the lower extremity. Surg Gynecol Obstet 1975; 140(2): 2468.

73. Collins TC, Johnson M, Daley J, Henderson WG, Khuri S, Gordon HS. Preoperative risk factors for 30-day mortality after elective surgery of vascular disease in Department of Veterans Affairs hospitals: is race important? J Vasc Surg 2001; 34(4): 634-40.

74. Axelrod DA, Upchurch GR Jr, DeMonner S, Stanley JC, Khuri S, Daley J, HendersonWG, Hayward R. Perioperative cardiovascular risk stratification of patients with diabetes who undergo elective major vascular surgery. J Vasc Surg 2002; 35(5): 894-901.

75. Menzoian JO, Sequeira JC, Doyle JE, Cantelmo NL, Nowak M, Tracey K, Zimmerman R, Mozden PJ. Therapeutic and clinical course of deep vein thrombosis. Am J Surg 1983; 146(5): 581-5.

76. Mattos MA, Melendres G, Sumner DS, Hood DB, Barkmeier LD, Hodgson KJ, Ramsey DE. Prevalence and distribution of calf vein thrombosis in 
patients with symptomatic deep venous thrombosis: a color-flow duplex study. J Vasc Surg 1996; 24(5): 738-44.

77. Fink AM, Mayer W, Steiner A. Extent of thrombus evaluated in patients with recurrent and first deep vein thrombosis. J Vasc Surg 2002; 36(2): 35760.

78. Meissner MH, Caps MT, Bergelin RO, Manzo RA, Strandness DE Jr. Early outcome after isolated calf vein thrombosis. J Vasc Surg 1997;26(5): 74956.

79. Heit JA, Silverstein MD, Mohr DN, Petterson TM, O'Fallon WN, Melton LJ 3rd. Risk factors for deep vein thrombosis and pulmonary embolism: a population-based case-control study. Arch Intern Med 2000; 160(6): 809-15. 
9. APÊNDICE 


\section{Articles in Press}

Incidence of ipsilateral postoperative deep venous thrombosis in the amputated lower extremity of patients with peripheral obstructive arterial disease

Marcelo Fernando Matielo, MD酗 $\square$, Calógero Presti, MD, PhD, Ivan Benaduce Casella, MD, Baptista Muraco Netto, MD, PhD, Pedro PuechLeão, MD, PhD

Received 28 April 2008; accepted 15 July 2008. published online 02 October 2008.

Corrected Proof

\section{Objective}

Patients undergoing amputation of the lower limb due to peripheral arterial disease (PAD) are at risk of developing deep venous thrombosis (DVT). Few studies in the research literature report the incidence of DVT during the early postoperative period or the risk factors for the development of DVT in the amputation stump. This prospective study evaluated the incidence of DVT during the first 35 postoperative days in patients who had undergone amputation of the lower extremity due to PAD and its relation to comorbidities and death.

\section{Methods}

Between September 2004 and March 2006, 56 patients (29 men), with a mean age of 67.25 years, underwent 62 amputations, comprising 36 below knee amputations (BKA) and 26 above knee amputations (AKA). EchoDoppler scanning was performed preoperatively and on postoperative days 7 and 31 (approximately). All patients received acetylsalicylic acid (100 mg daily) preoperatively and postoperatively, but none received prophylactic anticoagulation.

\section{Results}

DVT occurred in $25.8 \%$ of extremities with amputations (10 AKA and 6 BKA). The cumulative incidence in the 35 -day postoperative period was $28 \%$ (Kaplan-Meier). There was a significant difference $(P=.04)$ in the incidence of DVT between AKA (37.5\%) and BKA (21.2\%). Age $\geq 70$ years (48.9\% vs $16.8 \%, P=.021$ ) was also a risk factor for DVT in the univariate analysis. Of 
the 16 cases, $14(87.5 \%)$ were diagnosed during outpatient care. The time to discharge after amputation was averaged 6.11 days in-hospital stay (range, 1-56 days). One symptomatic nonfatal pulmonary embolism occurred in a patient already diagnosed with DVT. There was no relation between other comorbidities and DVT. The multivariate analysis showed no association between risk factors and the occurrence of DVT in the amputated extremity. DVT ipsilateral to the amputation did not influence the mortality rate $(9.7 \%)$.

\section{Conclusion}

The incidence of DVT in the early postoperative period ( $\leq 35$ days) was elevated principally in patients aged $\geq 70$ years and for AKA. Patients with PAD who have recently undergone major amputations should be considered at high risk for DVT, even after hospital discharge. Given the high rate of postoperative DVT observed in this study, we now recommend prophylactic anticoagulation for these patients, but further study is needed to determine the optimal duration and efficacy of this treatment.

Division of Vascular and Endovascular Surgery, Clinics Hospital of the Faculty of Medicine, São Paulo University, São Paulo, Brazil

国 Reprint requests: Marcelo Fernando Matielo, R. Dr. Neto de Araújo, 320 cj 1007, 04111-001 São Paulo-SP, Brazil

Competition of interest: none.

PII: S0741-5214(08)01193-2

doi:10.1016/j.jvs.2008.07.055

(C) 2008 Published by Elsevier Inc. 


\section{Resumo dos dados coletados}

\begin{tabular}{|c|c|c|c|c|c|c|c|c|c|c|c|c|c|c|c|c|c|c|c|}
\hline 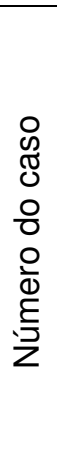 & 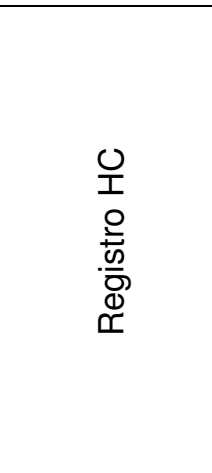 & 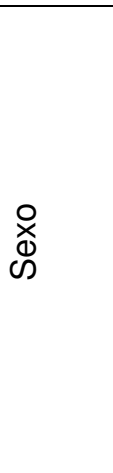 & $\begin{array}{l}\frac{0}{0} \\
\frac{\pi}{0}\end{array}$ & 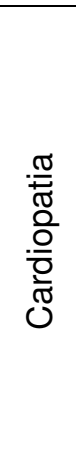 & $\begin{array}{l}\frac{0}{0} \\
\frac{\pi}{0} \\
\frac{0}{0} \\
0 \\
0\end{array}$ & $\begin{array}{l}\stackrel{\mathscr{N}}{N} \\
\stackrel{N}{\mathbb{N}} \\
>\end{array}$ & 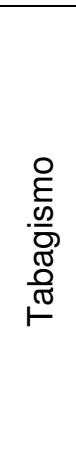 & 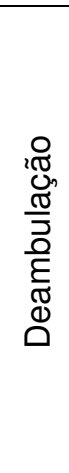 & $\underset{1}{\infty}$ & $\stackrel{\bigcup}{\gtrless}$ & $\sum_{\Delta}$ & 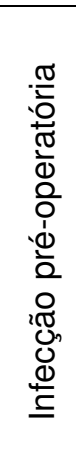 & 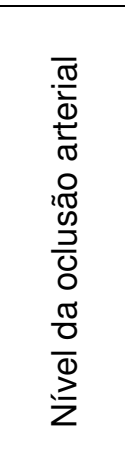 & 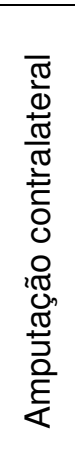 & 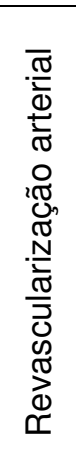 & 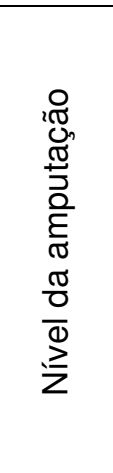 & 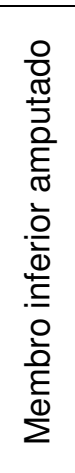 & 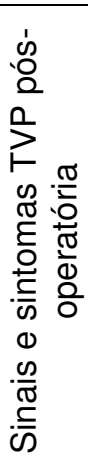 & 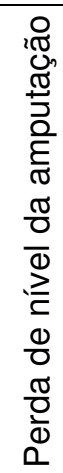 \\
\hline 1 & $13640862 \mathrm{~A}$ & masc & 82 & $\operatorname{sim}$ & nao & nao & nao & $\operatorname{sim}$ & sim & nao & $\operatorname{sim}$ & sim & popdis & nao & nao & perna & esq & nao & nao \\
\hline 2 & 13648595-E & fem & 75 & nao & nao & nao & nao & nao & $\operatorname{sim}$ & sim & nao & sim & fepo & nao & nao & coxa & esq & nao & nao \\
\hline 3 & 13648598-B & fem & 76 & nao & nao & nao & nao & nao & sim & nao & nao & $\operatorname{sim}$ & fepo & nao & nao & coxa & dir & $\operatorname{sim}$ & nao \\
\hline 4 & 13585117-H & fem & 65 & nao & nao & nao & nao & $\mathrm{sim}$ & sim & nao & sim & sim & fepo & sim & nao & perna & esq & nao & nao \\
\hline 5 & $13652632-B$ & masc & 54 & nao & nao & nao & nao & $\operatorname{sim}$ & nao & nao & $\operatorname{sim}$ & sim & popdis & nao & nao & coxa & esq & $\operatorname{sim}$ & nao \\
\hline 7 & 12.480 .617 & masc & 82 & sim & nao & nao & nao & nao & nao & $\operatorname{sim}$ & nao & $\operatorname{sim}$ & fepo & nao & nao & coxa & esq & $\operatorname{sim}$ & nao \\
\hline 8 & 19451931 & fem & 62 & nao & nao & nao & nao & nao & sim & nao & $\operatorname{sim}$ & $\operatorname{sim}$ & fepo & nao & nao & perna & esq & nao & nao \\
\hline 12 & $13659370 \mathrm{~B}$ & masc & 46 & nao & $\operatorname{sim}$ & nao & nao & $\operatorname{sim}$ & $\operatorname{sim}$ & nao & $\operatorname{sim}$ & nao & fepo & nao & nao & perna & dir & $\operatorname{sim}$ & nao \\
\hline 14 & 13657004-h & masc & 60 & nao & nao & nao & nao & $\operatorname{sim}$ & $\operatorname{sim}$ & nao & sim & sim & popdis & nao & $\operatorname{sim}$ & perna & dir & nao & nao \\
\hline 15 & 13.555.944J & fem & 65 & nao & nao & nao & nao & $\operatorname{sim}$ & sim & nao & $\operatorname{sim}$ & sim & fepo & nao & nao & coxa & dir & $\operatorname{sim}$ & nao \\
\hline 16 & $13593465 i$ & masc & 57 & nao & nao & sim & nao & nao & $\operatorname{sim}$ & $\operatorname{sim}$ & $\operatorname{sim}$ & sim & fepo & nao & nao & perna & esq & nao & nao \\
\hline 17 & $13655648 \mathrm{k}$ & masc & 46 & nao & nao & nao & $\operatorname{sim}$ & $\operatorname{sim}$ & nao & nao & nao & sim & fepo & nao & nao & perna & dir & nao & $\operatorname{sim}$ \\
\hline 18 & 13654838 & fem & 75 & nao & nao & sim & nao & $\operatorname{sim}$ & sim & nao & $\operatorname{sim}$ & $\operatorname{sim}$ & fepo & nao & nao & perna & esq & nao & nao \\
\hline 19 & $13661934 i$ & fem & 55 & nao & sim & sim & nao & nao & $\operatorname{sim}$ & nao & sim & sim & fepo & nao & nao & perna & esq & nao & nao \\
\hline 20 & $2408729 E$ & masc & 81 & nao & nao & $\operatorname{sim}$ & nao & nao & $\operatorname{sim}$ & nao & $\operatorname{sim}$ & sim & fepo & nao & nao & perna & dir & nao & nao \\
\hline 22 & 13663484-D & fem & 43 & nao & nao & nao & nao & $\mathrm{sim}$ & nao & nao & $\operatorname{sim}$ & sim & aortoil & nao & nao & perna & dir & nao & nao \\
\hline 23 & $13655648 \mathrm{k}$ & masc & 46 & nao & nao & nao & $\operatorname{sim}$ & nao & nao & nao & nao & sim & fepo & nao & nao & coxa & dir & nao & nao \\
\hline 24 & $5134754 \mathrm{k}$ & fem & 85 & $\operatorname{sim}$ & nao & nao & nao & nao & sim & nao & $\operatorname{sim}$ & nao & fepo & nao & nao & perna & dir & nao & $\operatorname{sim}$ \\
\hline 28 & $3066748 \mathrm{e}$ & fem & 58 & nao & sim & sim & nao & nao & sim & sim & sim & $\operatorname{sim}$ & fepo & nao & nao & perna & esq & nao & sim \\
\hline 31 & 13669199k & masc & 64 & nao & nao & $\operatorname{sim}$ & nao & nao & nao & nao & $\operatorname{sim}$ & sim & popdis & nao & nao & perna & dir & $\operatorname{sim}$ & nao \\
\hline 32 & $1367099 i$ & fem & 67 & nao & nao & nao & nao & nao & sim & nao & $\operatorname{sim}$ & $\operatorname{sim}$ & popdis & nao & nao & perna & dir & nao & nao \\
\hline 33 & $13639849 e$ & fem & 55 & sim & nao & nao & nao & $\operatorname{sim}$ & sim & nao & sim & nao & aortoil & nao & nao & coxa & dir & sim & nao \\
\hline 34 & $13661357 b$ & fem & 61 & nao & nao & nao & nao & nao & $\operatorname{sim}$ & $\operatorname{sim}$ & $\operatorname{sim}$ & $\operatorname{sim}$ & fepo & nao & nao & coxa & dir & $\operatorname{sim}$ & nao \\
\hline
\end{tabular}




\section{Continuação}

\begin{tabular}{|c|c|c|c|c|c|c|c|c|c|c|c|c|}
\hline 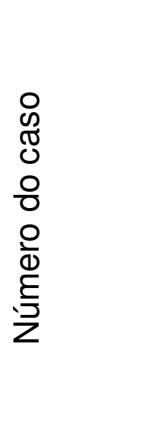 & 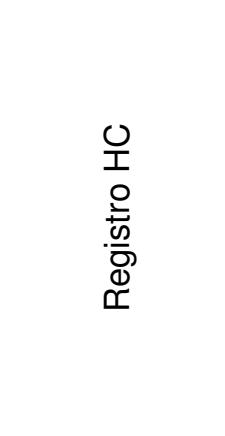 & 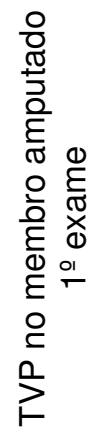 & 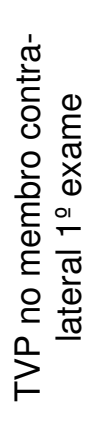 & 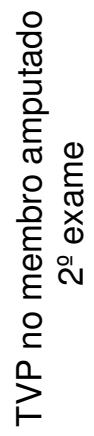 & 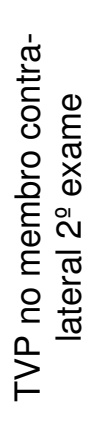 & 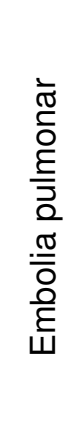 & 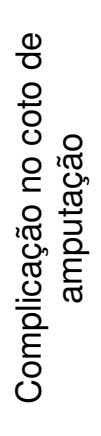 & 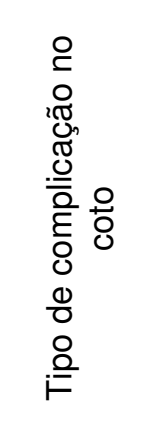 & 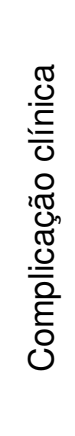 & $\frac{0}{\frac{1}{0}}$ & $\begin{array}{l}\frac{0}{0} \\
\frac{\pi}{0} \\
\frac{0}{2} \\
\frac{1}{0} \\
0 \\
0 \\
\frac{0}{0} \\
0 \\
\frac{\pi}{0}\end{array}$ & $\begin{array}{l}\frac{0}{0} \\
\frac{\pi}{0} \\
\frac{0}{2} \\
\frac{1}{0} \\
0\end{array}$ \\
\hline 1 & $13640862 A$ & nao & nao & nao & nao & nao & nao & & $\operatorname{sim}$ & nao & 25 & pervio \\
\hline 2 & 13648595-E & nao & nao & nao & nao & nao & nao & & sim & nao & 65 & pervio \\
\hline 3 & 13648598-B & $\operatorname{sim}$ & $\operatorname{sim}$ & $\operatorname{sim}$ & $\operatorname{sim}$ & nao & nao & & nao & nao & 3 & tvp \\
\hline 4 & 13585117-H & nao & nao & nao & nao & nao & $\operatorname{sim}$ & infeccao & nao & nao & 27 & pervio \\
\hline 5 & 13.652.632-B & $\operatorname{sim}$ & nao & nao & nao & nao & $\operatorname{sim}$ & infeccao & nao & nao & 7 & tvp \\
\hline 7 & 12.480 .617 & nao & nao & & & nao & nao & & $\operatorname{sim}$ & $\operatorname{sim}$ & 5 & pervio \\
\hline 8 & 19451931 & nao & nao & nao & nao & nao & nao & & nao & nao & 30 & pervio \\
\hline 12 & $13659370 \mathrm{~B}$ & nao & nao & nao & nao & nao & nao & & nao & nao & 27 & pervio \\
\hline 14 & 13657004-h & nao & nao & nao & nao & nao & $\operatorname{sim}$ & necrose & nao & nao & 30 & pervio \\
\hline 15 & $13.555 .944 \mathrm{~J}$ & $\operatorname{sim}$ & $\operatorname{sim}$ & $\operatorname{sim}$ & $\operatorname{sim}$ & nao & nao & & nao & nao & 13 & tvp \\
\hline 16 & $13593465 i$ & nao & nao & nao & nao & nao & $\operatorname{sim}$ & infeccao & nao & nao & 33 & pervio \\
\hline 17 & $13655648 \mathrm{k}$ & nao & nao & & & nao & $\operatorname{sim}$ & infeccao & nao & nao & 4 & pervio \\
\hline 18 & 13654838 & nao & nao & nao & nao & nao & $\operatorname{sim}$ & infeccao & nao & nao & 31 & pervio \\
\hline 19 & $13661934 \mathrm{i}$ & nao & nao & $\operatorname{sim}$ & nao & nao & $\operatorname{sim}$ & infeccao & nao & nao & 31 & tvp \\
\hline 20 & $2408729 E$ & nao & nao & nao & nao & nao & $\operatorname{sim}$ & infeccao & nao & nao & 33 & pervio \\
\hline 22 & 13663484-D & nao & nao & nao & nao & nao & $\operatorname{sim}$ & infeccao & nao & nao & 68 & pervio \\
\hline 23 & $13655648 \mathrm{k}$ & nao & nao & nao & nao & nao & nao & & nao & nao & 32 & pervio \\
\hline 24 & $5134754 \mathrm{k}$ & nao & nao & & & nao & $\operatorname{sim}$ & infeccao & nao & nao & 3 & pervio \\
\hline 28 & $3066748 \mathrm{e}$ & nao & nao & nao & nao & nao & $\operatorname{sim}$ & infeccao & nao & nao & 31 & pervio \\
\hline 31 & $13669199 \mathrm{k}$ & nao & nao & nao & nao & nao & nao & & nao & nao & 31 & pervio \\
\hline 32 & $1367099 i$ & nao & nao & nao & nao & nao & nao & & nao & nao & 30 & pervio \\
\hline 33 & $13639849 e$ & nao & nao & $\operatorname{sim}$ & nao & nao & $\operatorname{sim}$ & infeccao & nao & nao & 36 & tvp \\
\hline 34 & $13661357 b$ & nao & nao & nao & nao & nao & nao & & nao & nao & 28 & pervio \\
\hline
\end{tabular}




\section{Continuação}

\begin{tabular}{|c|c|c|c|c|c|c|c|c|c|c|c|c|c|c|c|c|c|c|c|}
\hline 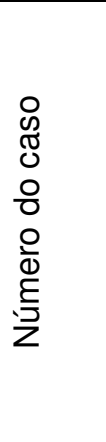 & 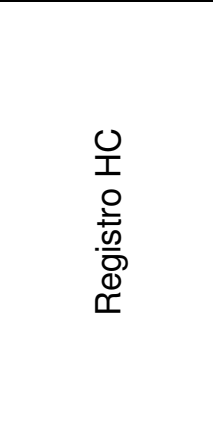 & $\begin{array}{l}\text { O } \\
\text { ભ } \\
\text { ஸे }\end{array}$ & $\begin{array}{l}\text { 0 } \\
\text { ㅁ } \\
\text { ㅇ }\end{array}$ & $\begin{array}{l}\stackrel{\frac{\pi}{J}}{\mathbb{N}} \\
\frac{O}{0} \\
\frac{0}{0} \\
\frac{\mathbb{J}}{J}\end{array}$ & $\begin{array}{l}\frac{0}{0} \\
\frac{\pi}{0} \\
\frac{0}{0} \\
0 \\
0\end{array}$ & $\begin{array}{l}\mathscr{D} \\
\stackrel{\mathbb{N}}{*} \\
\stackrel{\mathbb{N}}{>}\end{array}$ & 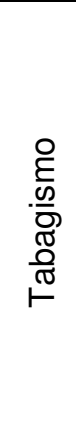 & 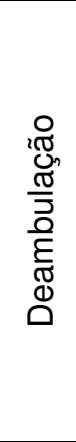 & 足 & $\stackrel{\bigcup}{\gtrless}$ & $\sum_{0}$ & 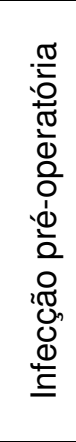 & 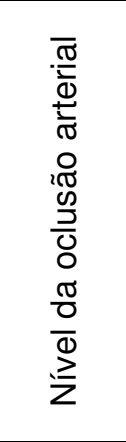 & 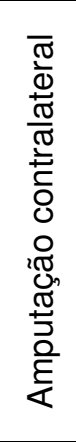 & 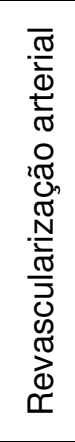 & 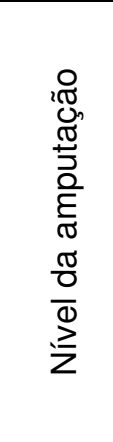 & 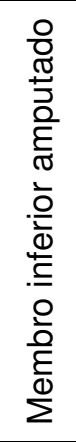 & 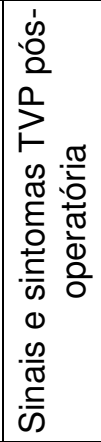 & 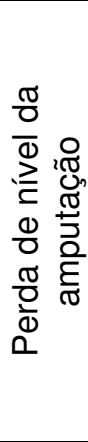 \\
\hline 36 & $3204347 j$ & fem & 45 & nao & nao & nao & nao & nao & $\operatorname{sim}$ & nao & $\operatorname{sim}$ & $\operatorname{sim}$ & fepo & nao & nao & perna & esq & nao & nao \\
\hline 37 & $3066748 \mathrm{e}$ & fem & 58 & nao & $\operatorname{sim}$ & $\operatorname{sim}$ & nao & nao & $\operatorname{sim}$ & sim & sim & $\operatorname{sim}$ & fepo & nao & nao & coxa & esq & $\operatorname{sim}$ & nao \\
\hline 38 & $13672804 \mathrm{k}$ & masc & 88 & sim & nao & $\operatorname{sim}$ & nao & $\operatorname{sim}$ & $\operatorname{sim}$ & nao & nao & $\operatorname{sim}$ & fepo & nao & nao & coxa & esq & $\operatorname{sim}$ & nao \\
\hline 39 & 13673694 & masc & 58 & $\operatorname{sim}$ & $\operatorname{sim}$ & nao & nao & nao & $\operatorname{sim}$ & $\operatorname{sim}$ & nao & nao & popdis & nao & nao & perna & esq & nao & nao \\
\hline 43 & 13681048-d & fem & 85 & nao & nao & nao & nao & nao & $\operatorname{sim}$ & nao & sim & $\operatorname{sim}$ & fepo & nao & nao & coxa & esq & nao & nao \\
\hline 44 & $13503761-x$ & fem & 67 & nao & nao & nao & nao & nao & $\operatorname{sim}$ & nao & sim & sim & fepo & nao & nao & perna & dir & nao & nao \\
\hline 45 & $13680948-F$ & masc & 72 & $\operatorname{sim}$ & nao & nao & nao & nao & $\operatorname{sim}$ & nao & $\operatorname{sim}$ & $\operatorname{sim}$ & aortoil & nao & $\operatorname{sim}$ & perna & dir & $\operatorname{sim}$ & nao \\
\hline 50 & $13682897 \mathrm{~h}$ & masc & 75 & nao & nao & sim & sim & nao & nao & nao & nao & nao & fepo & nao & nao & perna & esq & nao & nao \\
\hline 51 & $1353568 f$ & masc & 63 & nao & nao & $\operatorname{sim}$ & sim & $\operatorname{sim}$ & nao & nao & sim & $\operatorname{sim}$ & popdis & nao & nao & perna & dir & nao & nao \\
\hline 52 & $9.158 .140-3$ & masc & 76 & nao & $\operatorname{sim}$ & nao & nao & nao & $\operatorname{sim}$ & $\operatorname{sim}$ & sim & nao & fepo & nao & nao & coxa & esq & $\operatorname{sim}$ & nao \\
\hline 53 & 13682630-h & masc & 74 & sim & nao & nao & nao & nao & $\operatorname{sim}$ & $\operatorname{sim}$ & $\operatorname{sim}$ & sim & fepo & nao & nao & perna & esq & nao & $\operatorname{sim}$ \\
\hline 54 & $13691303 c$ & masc & 81 & sim & nao & nao & nao & nao & $\operatorname{sim}$ & nao & nao & $\operatorname{sim}$ & aortoil & nao & $\operatorname{sim}$ & perna & esq & nao & nao \\
\hline 56 & $13695468 \mathrm{k}$ & masc & 85 & sim & nao & nao & $\operatorname{sim}$ & $\operatorname{sim}$ & $\operatorname{sim}$ & $\operatorname{sim}$ & nao & nao & fepo & nao & nao & coxa & dir & nao & nao \\
\hline 57 & $3025932 b$ & masc & 85 & nao & nao & $\operatorname{sim}$ & nao & $\operatorname{sim}$ & $\operatorname{sim}$ & nao & nao & $\operatorname{sim}$ & fepo & $\operatorname{sim}$ & nao & perna & dir & nao & nao \\
\hline 58 & $3277834 \mathrm{e}$ & fem & 51 & nao & nao & nao & nao & $\operatorname{sim}$ & $\operatorname{sim}$ & nao & $\operatorname{sim}$ & sim & fepo & $\operatorname{sim}$ & nao & perna & esq & nao & nao \\
\hline 59 & $13698815 i$ & fem & 62 & nao & nao & nao & nao & nao & $\operatorname{sim}$ & nao & $\operatorname{sim}$ & sim & aortoil & nao & nao & perna & esq & nao & nao \\
\hline 60 & $13698815 i$ & fem & 62 & nao & nao & nao & nao & nao & $\operatorname{sim}$ & nao & sim & $\operatorname{sim}$ & fepo & nao & nao & perna & dir & nao & nao \\
\hline 61 & $13547647 d$ & masc & 63 & nao & nao & nao & $\operatorname{sim}$ & $\operatorname{sim}$ & nao & nao & nao & $\operatorname{sim}$ & fepo & $\operatorname{sim}$ & $\operatorname{sim}$ & coxa & esq & nao & nao \\
\hline 62 & $4061548 \mathrm{~h}$ & masc & 79 & nao & nao & nao & nao & nao & $\operatorname{sim}$ & $\operatorname{sim}$ & nao & nao & fepo & nao & nao & coxa & esq & nao & nao \\
\hline 63 & $2753599 \mathrm{j}$ & masc & 59 & nao & nao & nao & sim & $\operatorname{sim}$ & $\operatorname{sim}$ & nao & nao & sim & fepo & nao & $\operatorname{sim}$ & perna & esq & $\operatorname{sim}$ & nao \\
\hline 64 & $13679920 \mathrm{c}$ & masc & 71 & nao & nao & nao & $\operatorname{sim}$ & nao & $\operatorname{sim}$ & nao & $\operatorname{sim}$ & nao & aortoil & nao & nao & coxa & dir & nao & nao \\
\hline 65 & $13639849 e$ & fem & 56 & sim & nao & nao & nao & nao & $\operatorname{sim}$ & nao & $\operatorname{sim}$ & nao & aortoil & $\operatorname{sim}$ & nao & coxa & esq & nao & nao \\
\hline 67 & $13697980 \mathrm{e}$ & fem & 62 & sim & sim & sim & sim & nao & $\operatorname{sim}$ & $\operatorname{sim}$ & $\operatorname{sim}$ & $\operatorname{sim}$ & fepo & nao & nao & perna & dir & nao & nao \\
\hline
\end{tabular}




\section{Continuação}

\begin{tabular}{|c|c|c|c|c|c|c|c|c|c|c|c|c|}
\hline 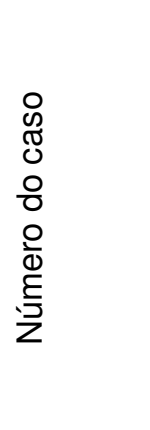 & 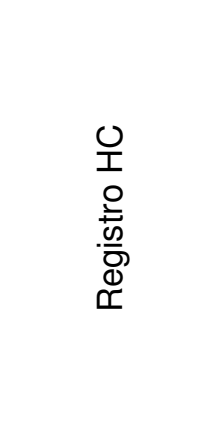 & 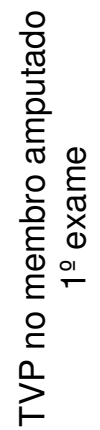 & 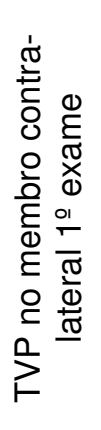 & 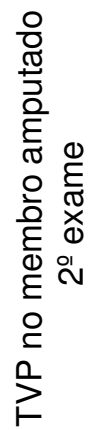 & 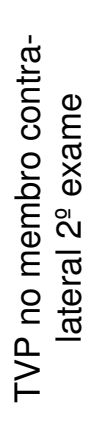 & 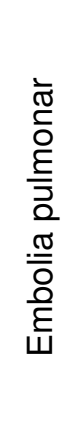 & 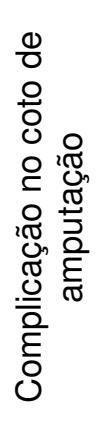 & 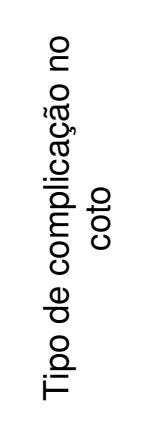 & 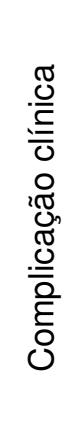 & $\frac{0}{\frac{1}{0}}$ & $\begin{array}{l}\frac{0}{0} \\
\frac{\pi}{0} \\
\frac{0}{2} \\
\frac{1}{0} \\
0 \\
0 \\
\frac{0}{0} \\
0 \\
\frac{\pi}{0}\end{array}$ & $\begin{array}{l}\frac{0}{0} \\
\frac{\pi}{0} \\
\frac{d}{2} \\
\frac{1}{0} \\
0\end{array}$ \\
\hline 36 & $3204347 j$ & nao & nao & nao & nao & nao & nao & & nao & nao & 26 & pervio \\
\hline 37 & $3066748 \mathrm{e}$ & $\operatorname{sim}$ & nao & $\operatorname{sim}$ & nao & nao & sim & infeccao & nao & nao & 3 & tvp \\
\hline 38 & $13672804 \mathrm{k}$ & $\operatorname{sim}$ & nao & nao & nao & nao & nao & & nao & nao & 3 & tvp \\
\hline 39 & 13673694 & nao & nao & nao & nao & nao & $\operatorname{sim}$ & mista & nao & nao & 29 & pervio \\
\hline 43 & $13681048-d$ & nao & nao & & & nao & nao & & nao & nao & 4 & pervio \\
\hline 44 & $13503761-x$ & nao & nao & nao & nao & nao & nao & & nao & nao & 33 & pervio \\
\hline 45 & 13680948-F & nao & nao & $\operatorname{sim}$ & nao & nao & $\operatorname{sim}$ & necrose & nao & nao & 26 & tvp \\
\hline 50 & $13682897 \mathrm{~h}$ & nao & nao & nao & nao & nao & nao & & nao & nao & 29 & pervio \\
\hline 51 & $1353568 f$ & nao & nao & nao & nao & nao & nao & & nao & nao & 29 & pervio \\
\hline 52 & $9.158 .140-3$ & nao & nao & nao & nao & nao & nao & & sim & sim & 33 & pervio \\
\hline 53 & 13682630-h & $\operatorname{sim}$ & $\operatorname{sim}$ & & & nao & $\operatorname{sim}$ & infeccao & $\operatorname{sim}$ & $\operatorname{sim}$ & 3 & tvp \\
\hline 54 & $13691303 c$ & nao & nao & nao & nao & nao & $\operatorname{sim}$ & necrose & nao & nao & 31 & pervio \\
\hline 56 & $13695468 \mathrm{k}$ & nao & nao & $\operatorname{sim}$ & nao & nao & nao & & nao & nao & 26 & tvp \\
\hline 57 & $3025932 b$ & nao & nao & sim & nao & nao & nao & & nao & nao & 14 & tvp \\
\hline 58 & $3277834 \mathrm{e}$ & nao & nao & nao & nao & nao & nao & & nao & nao & 29 & pervio \\
\hline 59 & $13698815 i$ & nao & nao & nao & nao & nao & $\operatorname{sim}$ & necrose & $\operatorname{sim}$ & nao & 31 & pervio \\
\hline 60 & $13698815 i$ & nao & nao & nao & nao & nao & sim & necrose & $\operatorname{sim}$ & nao & 31 & pervio \\
\hline 61 & $13547647 d$ & nao & nao & nao & nao & nao & nao & & nao & nao & 31 & pervio \\
\hline 62 & $4061548 \mathrm{~h}$ & $\operatorname{sim}$ & nao & & & nao & nao & & nao & nao & 9 & tvp \\
\hline 63 & $2753599 j$ & nao & nao & nao & nao & nao & nao & & nao & nao & 35 & pervio \\
\hline 64 & $13679920 \mathrm{c}$ & nao & nao & nao & nao & nao & nao & & nao & nao & 30 & pervio \\
\hline 65 & $13639849 \mathrm{e}$ & nao & nao & nao & nao & nao & nao & & nao & nao & 32 & pervio \\
\hline 67 & $13697980 \mathrm{e}$ & nao & nao & nao & nao & nao & $\operatorname{sim}$ & necrose & $\operatorname{sim}$ & nao & 36 & pervio \\
\hline
\end{tabular}




\section{Continuação}

\begin{tabular}{|c|c|c|c|c|c|c|c|c|c|c|c|c|c|c|c|c|c|c|c|}
\hline 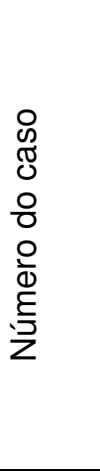 & 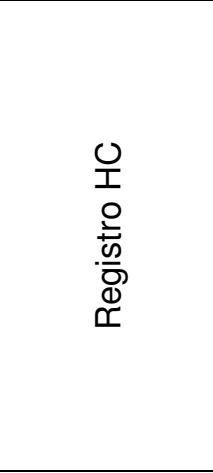 & $\begin{array}{l}\text { X } \\
\text { ஸ }\end{array}$ & $\begin{array}{l}\text { 음 } \\
\text { 으 }\end{array}$ & 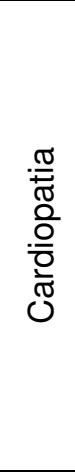 & $\begin{array}{l}\frac{0}{0} \\
\frac{\pi}{0} \\
\frac{0}{0} \\
0 \\
0\end{array}$ & 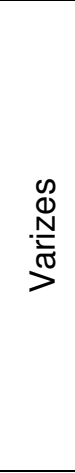 & 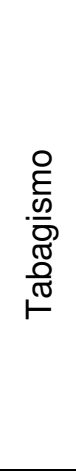 & 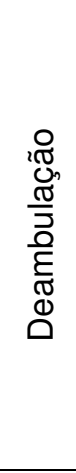 & $\underset{1}{\mathscr{X}}$ & $\stackrel{\bigcup}{\gtrless}$ & $\sum_{0}$ & 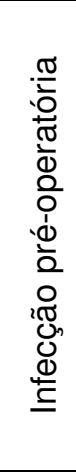 & 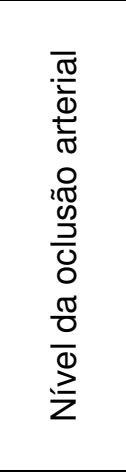 & 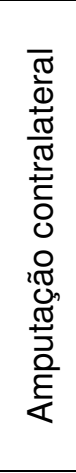 & 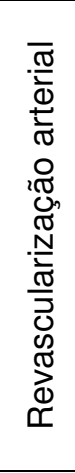 & 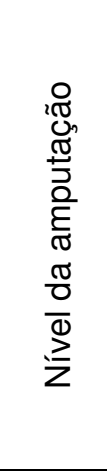 & 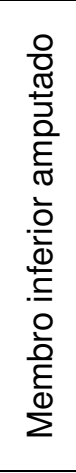 & 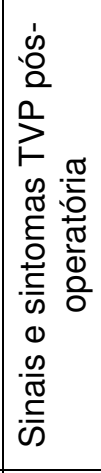 & 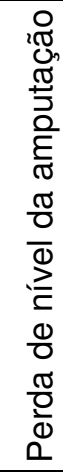 \\
\hline 68 & $13697980 \mathrm{e}$ & fem & 62 & sim & $\operatorname{sim}$ & $\operatorname{sim}$ & sim & nao & $\operatorname{sim}$ & sim & sim & sim & fepo & nao & nao & perna & esq & nao & nao \\
\hline 69 & $13697304 \mathrm{e}$ & fem & 82 & sim & nao & $\operatorname{sim}$ & nao & nao & $\operatorname{sim}$ & nao & $\operatorname{sim}$ & $\operatorname{sim}$ & fepo & nao & nao & coxa & dir & nao & nao \\
\hline 70 & $13706840 \mathrm{~g}$ & masc & 74 & nao & nao & nao & $\operatorname{sim}$ & nao & nao & nao & nao & $\operatorname{sim}$ & aortoil & $\operatorname{sim}$ & $\operatorname{sim}$ & perna & esq & nao & nao \\
\hline 71 & $2605827 d$ & masc & 56 & nao & nao & nao & $\operatorname{sim}$ & $\operatorname{sim}$ & nao & nao & nao & nao & fepo & nao & nao & coxa & esq & $\operatorname{sim}$ & nao \\
\hline 72 & $13708571 \mathrm{j}$ & masc & 80 & nao & nao & nao & nao & $\operatorname{sim}$ & $\operatorname{sim}$ & nao & $\operatorname{sim}$ & $\operatorname{sim}$ & popdis & nao & nao & perna & dir & nao & nao \\
\hline 74 & $13714448 \mathrm{~h}$ & fem & 65 & nao & nao & nao & nao & nao & $\operatorname{sim}$ & nao & $\operatorname{sim}$ & nao & aortoil & nao & $\operatorname{sim}$ & coxa & dir & nao & nao \\
\hline 76 & $13625365 e$ & masc & 60 & nao & nao & nao & nao & nao & nao & nao & $\operatorname{sim}$ & $\operatorname{sim}$ & fepo & nao & nao & coxa & dir & nao & nao \\
\hline 77 & $3171129 b$ & masc & 66 & nao & nao & nao & $\operatorname{sim}$ & $\operatorname{sim}$ & $\operatorname{sim}$ & nao & nao & nao & fepo & nao & nao & coxa & esq & $\operatorname{sim}$ & nao \\
\hline 78 & $30480082 \mathrm{~g}$ & masc & 69 & sim & nao & nao & nao & nao & $\operatorname{sim}$ & nao & nao & $\operatorname{sim}$ & fepo & nao & nao & coxa & dir & $\operatorname{sim}$ & nao \\
\hline 81 & $4006895 f$ & fem & 71 & nao & nao & nao & nao & nao & $\operatorname{sim}$ & nao & $\operatorname{sim}$ & sim & fepo & nao & nao & coxa & esq & nao & nao \\
\hline 82 & $3281537 e$ & fem & 67 & $\operatorname{sim}$ & nao & $\operatorname{sim}$ & nao & nao & $\operatorname{sim}$ & nao & $\operatorname{sim}$ & $\operatorname{sim}$ & popdis & nao & nao & perna & dir & nao & nao \\
\hline 83 & 55440150j & fem & 83 & $\operatorname{sim}$ & nao & nao & nao & nao & $\operatorname{sim}$ & $\operatorname{sim}$ & $\operatorname{sim}$ & $\operatorname{sim}$ & fepo & nao & nao & coxa & esq & nao & nao \\
\hline 85 & $13719342 d$ & fem & 78 & nao & nao & nao & nao & $\operatorname{sim}$ & $\operatorname{sim}$ & nao & $\operatorname{sim}$ & nao & fepo & nao & $\operatorname{sim}$ & perna & dir & nao & $\operatorname{sim}$ \\
\hline 86 & $13702221 E$ & fem & 61 & nao & nao & nao & nao & nao & $\operatorname{sim}$ & nao & $\operatorname{sim}$ & $\operatorname{sim}$ & fepo & nao & nao & perna & dir & nao & nao \\
\hline 87 & $13719342 d$ & fem & 78 & nao & nao & nao & nao & $\operatorname{sim}$ & $\operatorname{sim}$ & nao & sim & sim & fepo & nao & nao & coxa & dir & nao & nao \\
\hline 88 & 137231569 & masc & 70 & nao & nao & nao & nao & $\operatorname{sim}$ & $\operatorname{sim}$ & nao & $\operatorname{sim}$ & $\operatorname{sim}$ & fepo & nao & nao & perna & esq & $\operatorname{sim}$ & nao \\
\hline
\end{tabular}




\section{Continuação}

\begin{tabular}{|c|c|c|c|c|c|c|c|c|c|c|c|c|}
\hline 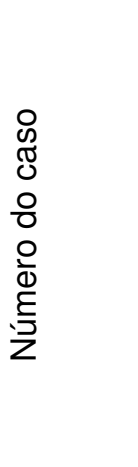 & 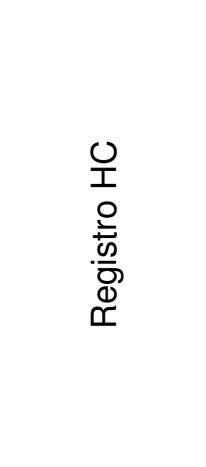 & 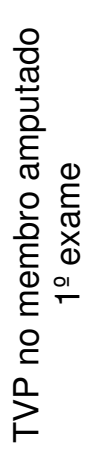 & 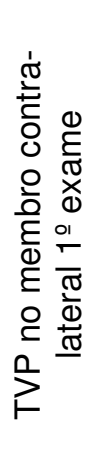 & 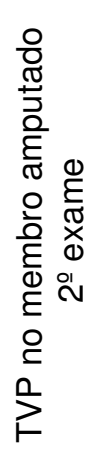 & 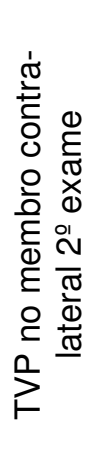 & 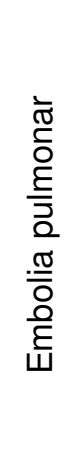 & 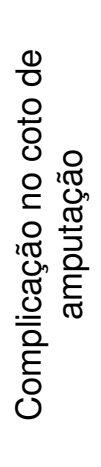 & 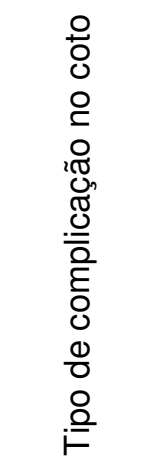 & 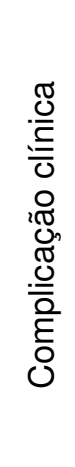 & $\begin{array}{l}\frac{0}{\overline{0}} \\
\frac{0}{0}\end{array}$ & $\begin{array}{l}\frac{0}{0} \\
\frac{\pi}{0} \\
\frac{0}{2} \\
\frac{1}{0} \\
0 \\
0 \\
\frac{1}{0} \\
\frac{\pi}{0} \\
\frac{\pi}{0}\end{array}$ & $\begin{array}{l}\frac{0}{0} \\
\frac{\pi}{0} \\
\frac{0}{2} \\
\frac{1}{0} \\
0\end{array}$ \\
\hline 68 & $13697980 \mathrm{e}$ & nao & nao & nao & nao & nao & $\operatorname{sim}$ & necrose & $\operatorname{sim}$ & nao & 36 & pervio \\
\hline 69 & $13697304 \mathrm{e}$ & $\operatorname{sim}$ & nao & & & nao & nao & & nao & nao & 13 & tvp \\
\hline 70 & $13706840 \mathrm{~g}$ & $\operatorname{sim}$ & nao & & & $\operatorname{sim}$ & $\operatorname{sim}$ & mista & $\operatorname{sim}$ & $\operatorname{sim}$ & 4 & tvp \\
\hline 71 & $2605827 d$ & $\operatorname{sim}$ & nao & & & nao & nao & & nao & nao & 3 & tvp \\
\hline 72 & $13708571 \mathrm{j}$ & nao & nao & & & nao & nao & & nao & $\operatorname{sim}$ & 9 & pervio \\
\hline 74 & 13714448h & nao & nao & nao & nao & nao & nao & & nao & nao & 34 & pervio \\
\hline 76 & $13625365 \mathrm{e}$ & nao & nao & nao & nao & nao & nao & & nao & nao & 26 & pervio \\
\hline 77 & $3171129 b$ & nao & nao & nao & nao & nao & nao & & nao & nao & 28 & pervio \\
\hline 78 & $30480082 \mathrm{~g}$ & nao & nao & nao & nao & nao & nao & & nao & nao & 30 & pervio \\
\hline 81 & $4006895 f$ & nao & nao & nao & nao & nao & nao & & nao & nao & 29 & pervio \\
\hline 82 & $3281537 e$ & nao & nao & nao & nao & nao & nao & & nao & nao & 28 & pervio \\
\hline 83 & 55440150j & nao & nao & & & nao & $\operatorname{sim}$ & mista & $\operatorname{sim}$ & $\operatorname{sim}$ & 7 & pervio \\
\hline 85 & $13719342 d$ & nao & nao & & & nao & $\operatorname{sim}$ & mista & nao & nao & 3 & pervio \\
\hline 86 & $13702221 \mathrm{E}$ & nao & nao & nao & nao & nao & sim & infeccao & nao & nao & 29 & pervio \\
\hline 87 & $13719342 d$ & nao & nao & nao & nao & nao & $\operatorname{sim}$ & infeccao & nao & nao & 28 & pervio \\
\hline 88 & 137231569 & $\operatorname{sim}$ & nao & & & nao & $\operatorname{sim}$ & infeccao & nao & nao & 9 & tvp \\
\hline
\end{tabular}

